\title{
Aeroacoustic and Flow Assessment of the Poro-Serrated Trailing Edges
}

\author{
Tze Pei Chong ${ }^{1}$, Elisa Dubois ${ }^{2}$, Alexandros Vathylakis ${ }^{3}$
}

Brunel University London, Uxbridge, UB8 3PH, UK

\begin{abstract}
This paper reports an aeroacoustic study of a NACA0012 aerofoil with a nonflat plate type serrated trailing edge, and a number of Poro-Serrated trailing edge devices that contain porous materials of various air flow resistances at the gaps between adjacent members of the serrated sawtooth. Free field noise measurements were performed inside an aeroacoustic wind tunnel facility, where the range of jet speeds was between $20 \mathrm{~ms}^{-1}$ and $60 \mathrm{~ms}^{-1}$. Flow measurements were also conducted in the same facility for the very near wake. The main objective of this work is to determine whether multiple-mechanisms on the broadband noise reduction can co-exist on a poro-serrated trailing edge. The results demonstrate that having low flow resistivity at the sawtooth gaps could not completely suppress the vortex shedding tone at low frequency, but it can achieve reasonably well broadband noise reduction at high frequency. With high flow resistivity at the sawtooth gaps, the denser porous material almost renders the poro-serrated trailing edge to becoming a straight trailing edge again, thus undermining the serration effect and causing a drop in the noise performance. The optimal range of flow resistivity for the poro-serrated trailing edge is found to be around $10 \mathrm{kN} . \mathrm{s.m}^{-4}$, where it can even perform slightly better than the conventional serrated trailing edge in terms of the turbulent broadband noise reduction while still completely suppresses the vortex shedding noise. From the analysis of the wake data, the overall drag force will not increase when a poro-serrated trailing edge is used.
\end{abstract}

\section{Introduction}

$\mathrm{E}^{\mathrm{n}}$ ngine jet noise used to be the major noise source for a civil aircraft. However, its dominance in aviation noise has been less significant since the introduction of high bypass ratio aero-engine. Nowadays, noise generated at the trailing edge of engine fan blades, or the airframe's high lift devices has become more important. The noise mechanism here is by the scattering of the hydrodynamic pressure fluctuations near the trailing edge. At high Reynolds numbers, the boundary layer develops over the aerofoil surface is turbulent and hence the radiated noise from the trailing edge is largely broadband in nature. The increase in air traffic leads to more frequent noise events to which airport neighbors are exposed to. The concern on the aircraft noise thus represents an important constraint on maintaining the growth in capacity of the airport transport system.

Another industrial application that utilises fan blades at high Reynolds number is the wind turbine, where trailing edge noise is one of the dominant noise sources. The swishing noise from a wind turbine blade is produced by the combination of trailing edge noise and Doppler amplification effect of the blade movement ${ }^{1}$. This swishing noise can be heard at a considerable distance, especially at low frequencies where atmospheric attenuation is not very

\footnotetext{
${ }^{1}$ Senior Lecturer, Department of Mechanical, Aerospace and Civil Engineering, t.p.chong@brunel.ac.uk, AIAA Member (corresponding author)

${ }^{2}$ Student, Department of Mechanical, Aerospace and Civil Engineering, elisa.dubois@gmail.com, Non-AIAA Member

${ }^{3}$ Research Associate, Department of Mechanical, Aerospace and Civil Engineering, alex.vat@hotmail.com, NonAIAA Member
} 
effective. This important environmental impact is one of the main reasons for the high rate of refusal to grant planning permission for onshore wind farm in many countries. Therefore, for the aero-engine, airframe and wind turbine industries, it is important to develop new or existing technologies to address the trailing edge noise problem.

Corrugating the trailing edges of fan blade in the form of serration is one of the promising methods to reduce trailing edge turbulent broadband noise ${ }^{2-7}$. This passive method has been demonstrated experimentally to achieve reduction of trailing edge turbulent broadband noise level up to $8 \mathrm{~dB}$. Serrated trailing edge is also effective in suppressing boundary layer instability tonal noise, where $20 \mathrm{~dB}$ in the Overall Sound Pressure Level reduction has been demonstrated ${ }^{8}$. Except in the works by ${ }^{8,9}$, most of the serrations have been formed from thin flat plates, which are inserted into the trailing edge of the main aerofoil body. This was done for ease of manufacture and, more importantly, to prevent vortex shedding arising from bluntness caused by cutting the serrations into the aerofoil main body. However, serrated flat plate inserts are unlikely to have the structural integrity for continuous operation at high loading configuration. Moreover, introducing flat plate inserts could alter the aerofoil geometry and hence the global circulation around the aerofoil is likely to be different from the original aerofoil. Another shortcoming of a flat plate serration is that the noise performance is susceptible to misalignment between the incoming flow angle and the serration flap angle ${ }^{5}$. It is also observed by some researchers that flat plate serration can cause an increase in noise level at high frequency ${ }^{3,5}$. All these factors could potentially prevent the eventual and widespread adoption of serration technology in the aircraft and wind turbine industries.

More preferable from the points of view of structural integrity and preserving the aerofoil shape is to cut the serration patterns directly into the aerofoil body. This configuration is henceforth referred to "nonflat plate serration". As shown in Fig. 1a, a nonflat plate serration can leave a certain degree of bluntness $(\varepsilon)$ on the sawtooth gaps. However, whilst the nonflat plate serration has also been shown to afford good level of broadband noise reduction across a wide frequency range ${ }^{9}$, the overall noise reduction is compromised by high level of bluntnessinduced narrowband vortex shedding noise predominantly at lower frequency. Previous attempts to reduce the bluntness-induced vortex shedding by wrapping woven-wire mesh screen around the nonflat plate serrated trailing edge was only partially successful ${ }^{10}$. The reason might be due to the low flow resistance of the mesh screen to be effective in suppressing the vortex shedding. The mesh screen also deteriorates the level of broadband noise reduction achieved by the serration. Moreover, noise level is found to increase at high frequency due to the surface roughness introduced by the mesh screen to the sawtooth surface. As a result, the overall noise performance is not improved much.

A more effective approach to introducing serrations directly into the main body of aerofoil is the "Poro-Serrated" concept $^{11}$, which utilises porous foams between adjacent members of the sawtooth to fill the air gap. As shown in Fig. 1b, these porous foams were cut precisely to match the exact volume and shape of the sawtooth gaps, so that an original aerofoil profile throughout the chord length is preserved. Whilst the porous foam at the sawtooth gaps has been shown to completely suppress the vortex shedding, significant turbulent broadband noise reduction can simultaneously be achieved. The paper also reports that for aerofoil with poro-serrated trailing edge, the lift and drag coefficients at the pre-stall regime remain largely the same performance as the baseline, straight trailing edge case.

So far, there is no general consensus regarding the exact mechanism of turbulent broadband noise reduction by a trailing edge serration. Theoretical approaches ${ }^{12,13}$ describe the obliqueness of the sawtooth edges as an effective geometry to reduce the coherences between the acoustic sources, and cause a phase shift in acoustic radiation along the wetted edge. Broadband noise reduction is therefore achieved through the destructive acoustical interference of these different modes. Results of an experimental study ${ }^{14}$ on turbulent flow over a flat plate with a serrated sawtooth trailing edge demonstrate that the wall pressure power spectral density and the spanwise coherence remain largely unchanged over large area of the sawtooth. However, they also observed a strong presence of pressure-driven oblique vortical structures near the sawtooth side edges, which interact with the turbulent boundary layer and cause a redistribution of the momentum transport and turbulence energy. It has been suggested that the vortical structure will cause a considerable effect on the acoustical scattering along the sawtooth oblique edges that eventually results in broadband noise reduction. However, further investigation is still needed to ascertain this.

It has also been demonstrated that turbulent broadband noise can be reduced when the whole trailing edge is made from porous materials ${ }^{15,16}$. The underpinning mechanism is perhaps more straightforward than the serration case. The porous nature of the material allows communication between flow on the suction and pressure sides, thus reduces the acoustical dipole strength at the trailing edge. An important parameter describing a porous material is the air flow resistivity, which is related to the amount of pressure drop across a porous material. Generally speaking, the level of the radiated broadband noise will be lower if the trailing edge has low flow resistivity so that transverse flow can be initiated at the trailing edge vicinity.

In the poro-serrated configuration, the main mechanism underpinning the broadband noise reduction is primarily associated with the serration geometry ${ }^{11}$. Although the porous material in this configuration is originally intended to 
(a) Nonflat plate serrated trailing edge

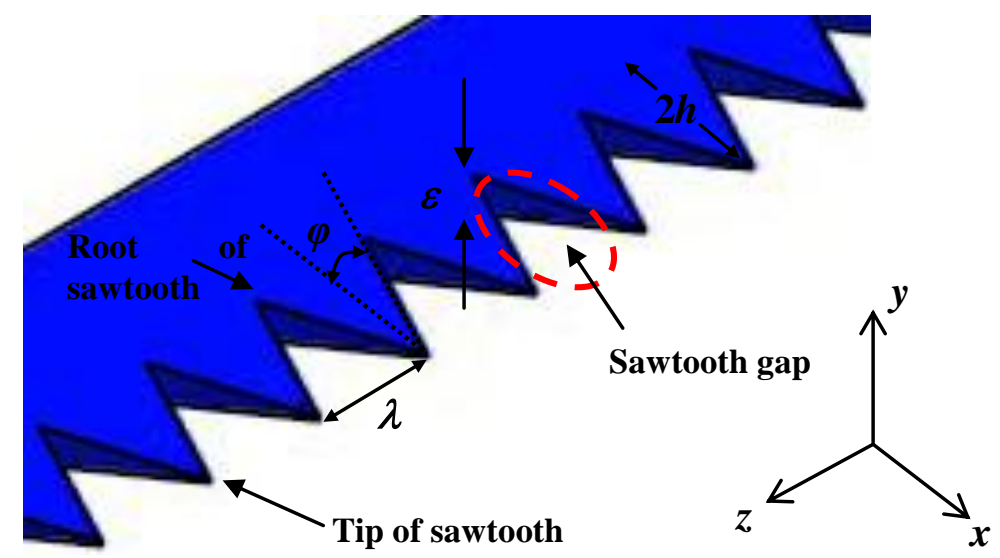

(b)

Poro-serrated trailing edge

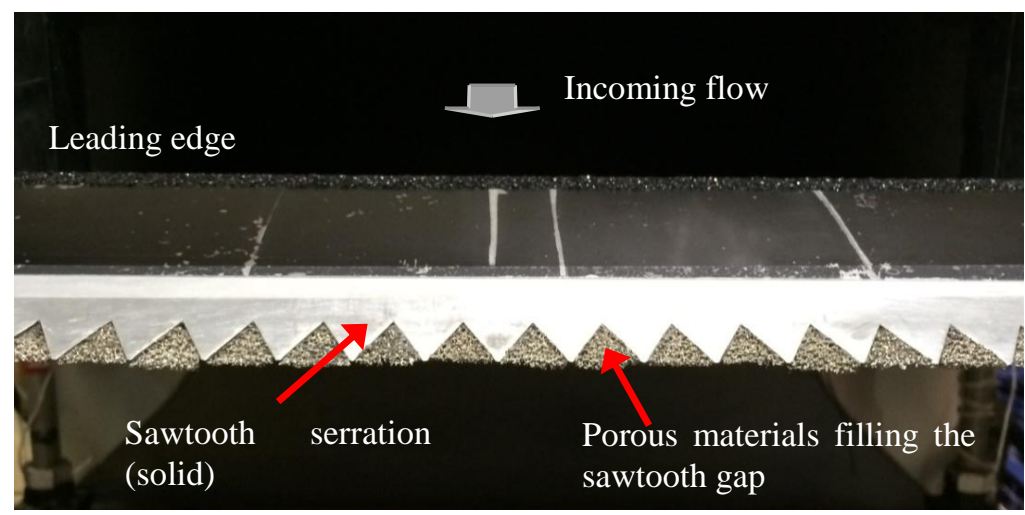

Fig. 1 (a) Parameters associated with a nonflat plate type sawtooth geometry: serration angle $(\varphi)$, serration length $(2 h)$, serration period $(\lambda)$ and root bluntness $(\varepsilon)$, (b) photograph of a poro-serrated trailing edge installed on a NACA0012.

eliminate the vortex shedding, the results in ${ }^{11}$ show that it also has a potential to contribute in further broadband noise reduction. It is therefore hypothesised that the poro-serrated trailing edge could offer further reduction in the broadband noise level.

The main objective of this work is to investigate whether multiple-broadband noise reduction mechanisms can co-exist in the poro-serrated trailing edge configuration. By utilising the same serration geometry throughout the aeroacoustic experiments, several porous materials that produce different flow resistivities were used to fill the sawtooth air gaps. The investigation also includes the nonflat plate configuration that comprises the same serration geometry but without the porous materials at the sawtooth air gaps.

\section{Experimental setup}

\section{A. Wind tunnel facilities and instrumentations}

Similar to the previous work in $^{8-11}$, the aerofoil under investigation is a NACA0012 aerofoil with a sawtooth serration cut directly into the main body of the aerofoil. The chord length $(C)$ of the aerofoil is $150 \mathrm{~mm}$, and the 


\begin{tabular}{|c|c|c|c|}
\hline Symbols & Descriptions & $\sigma\left(\mathrm{kN} . \mathrm{s} . \mathrm{m}^{-4}\right)$ & Main area(s) of study \\
\hline SO & Baseline, straight, nonporous solid trailing edge & $\infty$ & $\begin{array}{l}\text { Noise } \\
\text { Wake }\end{array}$ \\
\hline$S 1$ & $\begin{array}{c}\text { Nonflat plate serrated trailing edge; } \\
2 h=20 \mathrm{~mm}, \varphi=7^{\circ}, \lambda / h=0.49 \text { and } \varepsilon=5.7 \mathrm{~mm}\end{array}$ & 0 & Wake \\
\hline$S 1^{+}$ & $\begin{array}{c}\text { (Poro-Serrated trailing edge) } \\
2 h=20 \mathrm{~mm}, \varphi=7^{\circ}, \lambda / h=0.49 \text { and } \varepsilon=5.7 \mathrm{~mm} \\
\text { sawtooth gaps filled with Recemat }\end{array}$ & 8.0 & Wake \\
\hline$S 2$ & $\begin{array}{c}\text { Nonflat plate serrated trailing edge; } \\
2 h=20 \mathrm{~mm}, \varphi=10^{\circ}, \lambda / h=0.71 \text { and } \varepsilon=5.7 \mathrm{~mm}\end{array}$ & 0 & Noise \\
\hline$S 2^{I}$ & $\begin{array}{c}\text { (Poro-Serrated trailing edge) } \\
\text { Same serration parameters as S2; } \\
\text { sawtooth gaps filled with 45 PPI reticulated foam }\end{array}$ & 2.5 & Noise \\
\hline$S 2^{I I}$ & $\begin{array}{c}\text { (Poro-Serrated trailing edge) } \\
\text { Same serration parameters as S2; } \\
\text { sawtooth gaps filled with Basotect (Melamine) }\end{array}$ & 10 & Noise \\
\hline$S 2^{I I I}$ & $\begin{array}{c}\text { (Poro-Serrated trailing edge) } \\
\text { Same serration parameters as S2; } \\
\text { sawtooth gaps filled with Needle felting foam }\end{array}$ & 41.2 & Noise \\
\hline$S 2^{I V}$ & $\begin{array}{c}\text { (Poro-Serrated trailing edge) } \\
\text { Same serration parameters as S2; } \\
\text { sawtooth gaps filled with ArmaFoam Sound }\end{array}$ & 153.4 & Noise \\
\hline
\end{tabular}

Table 1 Summary of all the trailing edge devices tested in this study.

width is $300 \mathrm{~mm}$. Between the leading edge $x / C=0$, and $x / C=0.79$, the original NACA0012 aerofoil profile is unmodified, where $x$ is the streamwise direction. Further downstream, $0.79 \leq x / C \leq 1.0$, is a section that can be removed and replaced by either an unmodified or modified trailing edge profile. Once attached, the trailing edge section forms a continuous profile giving the appearance that the serrations are cut into the main body of the NACA0012 aerofoil. Figure 1a shows the parameters associated with a nonflat plate serrated trailing edge geometry. A prominent feature of this type of serrated trailing edge is the exposure of a significant bluntness $(\varepsilon)$ at the root region, which would otherwise be negligible for the conventional flat plate type serrated trailing edge. Schematic for a poro-serrated trailing edge is shown in Fig. 1b. As mentioned previously, the porous material was cut precisely to follow the volume and shape of each sawtooth gap to ensure that a continuous aerofoil profile is maintained.

Table 1 summarises the geometrical parameters of the trailing edge devices investigated in this study, including one with straight trailing edge $S 0$ to serve as the baseline case. The poro-serrated trailing edges $\left(S 2^{I}, S 2^{I I}, S 2^{I I I}\right.$ and $S 2^{I V}$ ) are based upon one particular nonflat plate serrated trailing edge geometry, $S 2$. Therefore they all share the same $2 h, \varphi, \lambda / h$ and $\varepsilon$, which should be a priori in the current optimisation study of the porous materials, except that the air flow resistivity at the sawtooth gaps is not the same because different types of porous materials were used. Throughout the paper, the flow resistivity at the sawtooth gaps is represented by $\sigma$. The values of $\sigma$ presented in the table were obtained experimentally from a purposely built system. The experimental procedure in determining the $\sigma$ will be discussed in Section II.B.

Likewise, another poro-serrated trailing edge $S 1^{+}$is based upon a nonflat plate serrated trailing edge geometry, $S 1$ that share the same $2 h, \varphi, \lambda / h$ and $\varepsilon$. The $S 1$ and $S 1^{+}$trailing edge devices were mainly used for the experiment in the very near wake measurement, although their noise characteristics have been reported elsewhere ${ }^{11}$.

Noise measurements were conducted in an aeroacoustic open jet wind tunnel at Brunel University London. The open jet wind tunnel is situated in a $4 \mathrm{~m} \times 5 \mathrm{~m} \times 3.4 \mathrm{~m}$ anechoic chamber to facilitate free field measurement of the aerofoil self-noise. As shown in Fig. 2, the nozzle exit is rectangular with dimensions of $0.10 \mathrm{~m}$ (height) x $0.30 \mathrm{~m}$ (width). This wind tunnel can achieve a turbulence intensity of between $0.1-0.2 \%$ and a maximum jet velocity of about $80 \mathrm{~ms}^{-1}$. The background noise of the wind tunnel facility is well below the self-noise of the quietest aerofoil 
across the whole range of velocity ${ }^{17}$. The range of jet speeds under investigation was between 20 and $60 \mathrm{~ms}^{-1}$, corresponding to Reynolds numbers based on $\mathrm{C}$ of $2 \times 10^{5}$ and $6 \times 10^{5}$ respectively. The aerofoil was held by side plates flushed with the nozzle lips. The aerofoil was set at $0^{\circ}$ angle of attack with relative to the jet flow direction.

As shown in Fig. 2, far field noise measurements were made by a single condenser microphone at polar angles of $\Theta=$ $90^{\circ}$ at a distance of $1.0 \mathrm{~m}$ from the aerofoil trailing edge at mid span. Noise data was acquired at a sampling frequency of 44 $\mathrm{kHz}$ for 10 seconds by a 16-bit Analogue-Digital card from National Instrument. The data was then windowed and the Power Spectral Density (PSD) of $1 \mathrm{~Hz}$ bandwidth was computed from a 1024 point FFT.

To investigate the footprints of the vortex shedding in the wake subjected to the poro-serrated trailing edge, single hot wire probe ( $5 \mu \mathrm{m}$ diameter, $1.25 \mathrm{~mm}$ length, DANTEC 55P11) was used to measure the mean and fluctuating velocities of the aerofoil wake at an overheat ratio of 1.8. Signals from the hot wire probe were digitised by a 12-bit A/D converter (TSI model ADCPCI) at a sampling frequency of $20 \mathrm{kHz}$ for 120000 realisations. The hot wire probe was attached to a computer-controlled two-dimensional traverse system with a resolution of $0.01 \mathrm{~mm}$ in both directions. In the current study, the velocity is measured in a $y-z$ plane at $x / C=1.03$ (for the coordinate system refer to Fig. 1).

\section{B. Air flow resistivity of the porous materials}

An important parameter for the poro-serrated trailing edge is the air flow resistivity of the porous materials and how they affect the noise reduction if incorporated in a trailing edge design. The definition of the flow resistivity of a porous sample subjected to through flow relates to the pressure difference $\Delta P$ on either side of the sample in question, to the thickness $t$ of the sample and the flow velocity $U$ :

$$
\sigma=\frac{\Delta P}{U t}
$$

Therefore measuring the pressure variation across a porous sample allows one to evaluate the flow resistivity. A simple rig was set up for this purpose. As illustrated in Fig. 3, the rig is essentially a $2 \mathrm{~m}$ long and $52 \mathrm{~mm}$ innerdiameter Perspex tube along which each porous sample can be inserted. A centrifugal fan is placed at one end of the

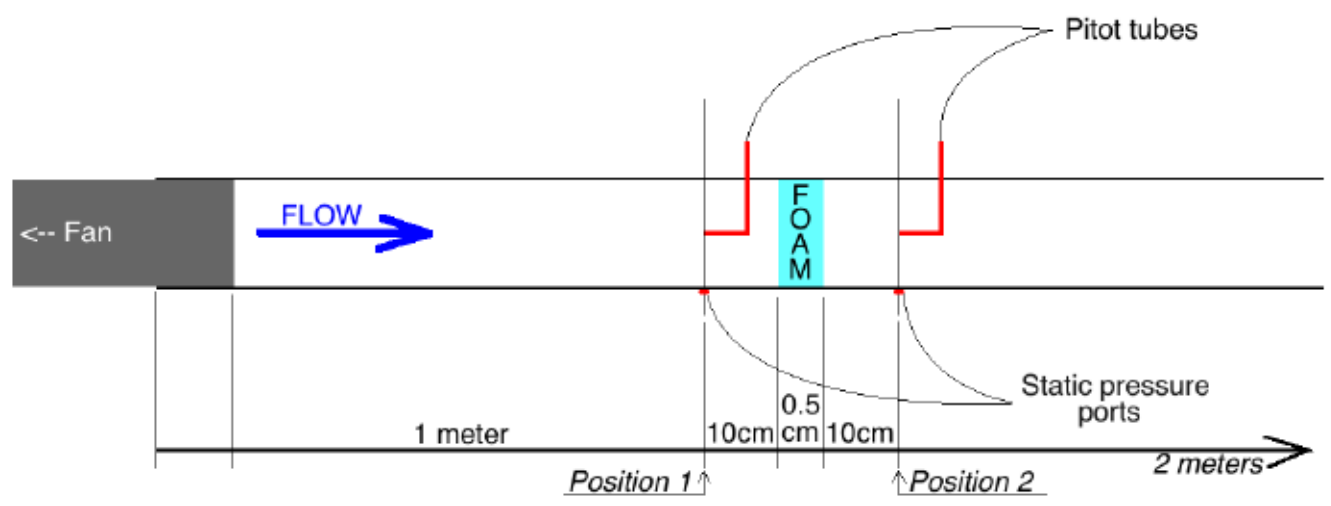

Fig. 3 Experimental set up for the measurement of air flow resistivity of different porous materials. 
(a)

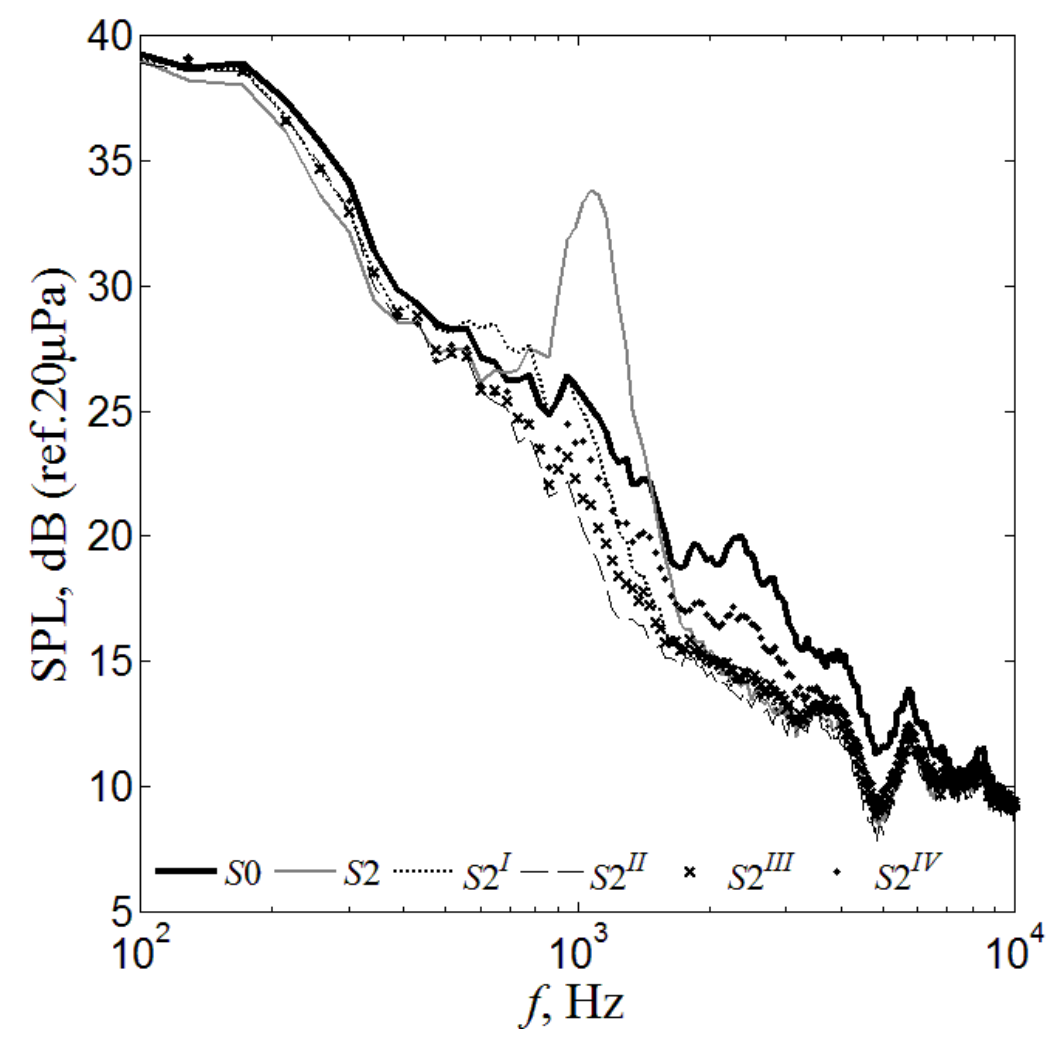

(b)

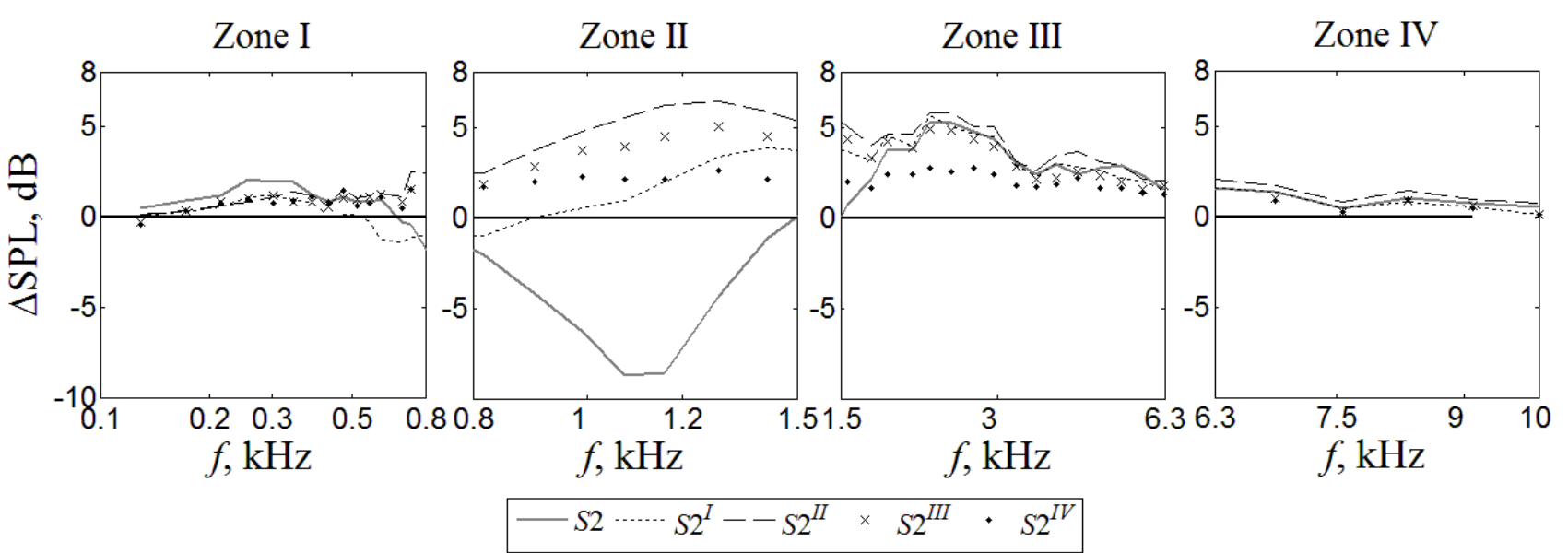

Fig. 4 Comparisons of (a) Sound Pressure Level (SPL), dB measured at $U=40 \mathrm{~ms}^{-1}$ for the $S 0$ (straight), $S 2$ (serrated), and $S 2^{I}, S 2^{I I}, S 2^{I I I}$ and $S 2^{I V}$ (poro-serrated) trailing edges, (b) $\Delta \mathrm{SPL}$, dB for the $S 2$ (serrated), and $S 2^{I}, S 2^{I I}, S 2^{I I I}$ and $S 2^{I V}$ (poro-serrated) trailing edges at frequency Zones I, II, III and IV.

tube to provide air flow of about $10 \mathrm{~ms}^{-1}$. The position of the porous sample with relative to the tube entrance was determined beforehand to ensure that it is placed after the internal flow is fully developed. The first static pressure tap of $0.5 \mathrm{~mm}$ diameter (upstream of the porous sample) was drilled at a position along the tube where the flow would be fully developed. Then, a hole that would allow the insertion of a Pitot tube was drilled further along the 
tube such that the Pitot tube tip and the static pressure tap on the Perspex tube would both be aligned at the same position (Position 1). The porous sample was then placed $10 \mathrm{~cm}$ away from that first pressure position. The second static pressure tap was drilled at the same $10 \mathrm{~cm}$ clearance from the aft region of the porous sample (Position 2). All the porous sample thickness was chosen as $5 \mathrm{~mm}$, which is close to the value of the serration bluntness $\varepsilon$.

The measured air flow resistivities for the four porous samples are listed in Table 1, which cover the range of $2<$ $\sigma<150 \mathrm{kN} . \mathrm{s} . \mathrm{m}^{-4}$. Note that these measured values are close to the values provided by the commercial suppliers of the porous material.

\section{General Characteristics of the Noise Spectra}

Using a single far field microphone at polar angle of $\Theta=90^{\circ}$ at a distance of $1.0 \mathrm{~m}$ from the aerofoil trailing edge at mid span, the Sound Pressure Level (SPL) produced by the six trailing edges are shown in Fig. 4a for velocity $U=40 \mathrm{~ms}^{-1}$. Although not included in the figure for clarity, the aerofoil with the quietest trailing edge still radiates larger level of self-noise than the background noise across the whole measured frequency range. Figure $4 \mathrm{~b}$ shows the difference in sound pressure level $(\triangle \mathrm{SPL})$ between the baseline aerofoil with straight trailing edge $S 0$ and the serrated trailing edges, which can be expressed as: $\triangle \mathrm{SPL}=\mathrm{SPL}_{(S 0)}-\mathrm{SPL}_{(S 2, S 2}{ }^{I-I V}{ }$. Therefore a positive $\Delta \mathrm{SPL}$ represents achievements in noise reduction, and a negative $\triangle \mathrm{SPL}$ is the opposite.

Figure $4 \mathrm{a}-\mathrm{b}$ demonstrates that trailing edge serration cut into the main body of the aerofoil $S 2$, or with the porous materials filling the gaps between adjacent members of the sawtooth $\left(S 2^{I}, S 2^{I I}, S 2^{I I I}\right.$ and $\left.S 2^{I V}\right)$, has a substantial effect on the radiated noise spectra compared to the baseline straight trailing edge $S 0$. For analysis purpose the acoustic spectra in Fig. $4 \mathrm{~b}$ are divided into four frequency $(f)$ zones. Zone I corresponds to low frequency band at $f \leq 800 \mathrm{~Hz}$. Zone II and Zone III encompass the frequency band at $800 \leq f \leq 1500 \mathrm{~Hz}$ and $1500 \leq f \leq 6300 \mathrm{~Hz}$, respectively. Finally, Zone IV represents the high frequency band at $f \geq 6300 \mathrm{~Hz}$. The following sub-sections discuss the characteristics and significances of the acoustic spectra at these different frequency zones.

\section{A. Zone I}

The acoustic spectra at Zone I are most likely to be contributed by the installation effects on the incoming jet. When the aerofoil is installed with the nonflat plate $S 2$ serrated trailing edge, positive $\Delta$ SPL in the region of $1.5-2$ $\mathrm{dB}$ is found across almost the entire frequency band within the Zone I in Fig. 4b. Such trend is highly repetitive because several confirmation tests essentially produced the same results. It should be emphasised that the seemingly "noise reduction" achieved by the $S 2$ serrated trailing edge at low frequency is not contributed by the serration effect per se, but rather it is likely to be caused by the slight change in global flow field around the aerofoil. It is believed that cutting any trailing edge serration pattern directly into the aerofoil body, such as the $S 2$ case, can cause a distortion of the rear stagnation points across the span of the aerofoil. Subsequently, jet flow approaching the leading edge cannot remain two-dimensional under which the front stagnation points and streamline across the span will vary according to the wavelength of the serrated trailing edge. The aerofoil might then experience different aerodynamic loading and, as a result, produces different level of noise radiation at low frequency compared to the baseline $S 0$ case.

The same trend in Zone I can be seen in the $\triangle$ SPL spectra pertaining to the $S 2^{I}, S 2^{I I}, S 2^{I I I}$ and $S 2^{I V}$ cases, although in this time the $+\Delta \mathrm{SPL}$ is lower at about $1 \mathrm{~dB}$. Unlike the $S 2$ nonflat plate serrated trailing edge, these poro-serrated trailing edges do not possess significant blunt portions at the rear part of the aerofoil because the sawtooth gaps have already been filled with porous material. As a result, the physical appearance of the poro-serrated trailing edges will resemble to the baseline $S 0$ straight trailing edge. However, the presence of the porous material can still instigate transverse flow near the trailing edge, thus distorting the rear stagnation points. Although less significant, the installation effects as experienced by the aerofoil subjected to the $S 2$ serrated trailing edge will also be present for the $S 2^{I}, S 2^{I I}, S 2^{I I I}$ and $S 2^{I V}$ poro-serrated trailing edges.

\section{B. Zone II}

The discussion now focuses on the frequency Zone II. A prominent feature here is the very large level of narrowband "tone" produced by the nonflat plate $S 2$ serration where the peak frequency occurs at about $1 \mathrm{kHz}$ producing large $\triangle \mathrm{SPL} \approx-9 \mathrm{~dB}$. Examining the acoustic spectra produced by the $S 2$ serration at other velocities indicates that the tone has a clear Strouhal number dependency $(f \varepsilon / U)$ of an averaged 0.158 , which is shown in Fig. 5a. The Strouhal number is defined with respect to the bluntness $\varepsilon(5.7 \mathrm{~mm})$ and flow velocity $U$. This suggests that the tone noise is associated with the periodic vortex shedding emanated from the sawtooth roots when almost no air 
(a)

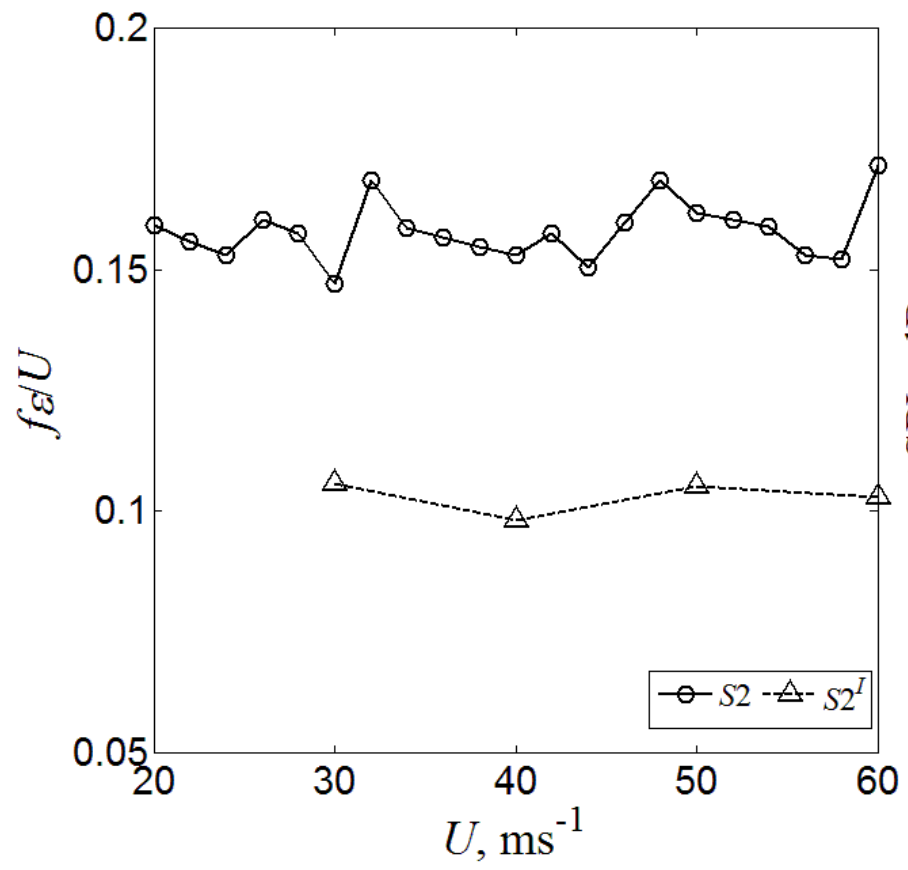

(b)

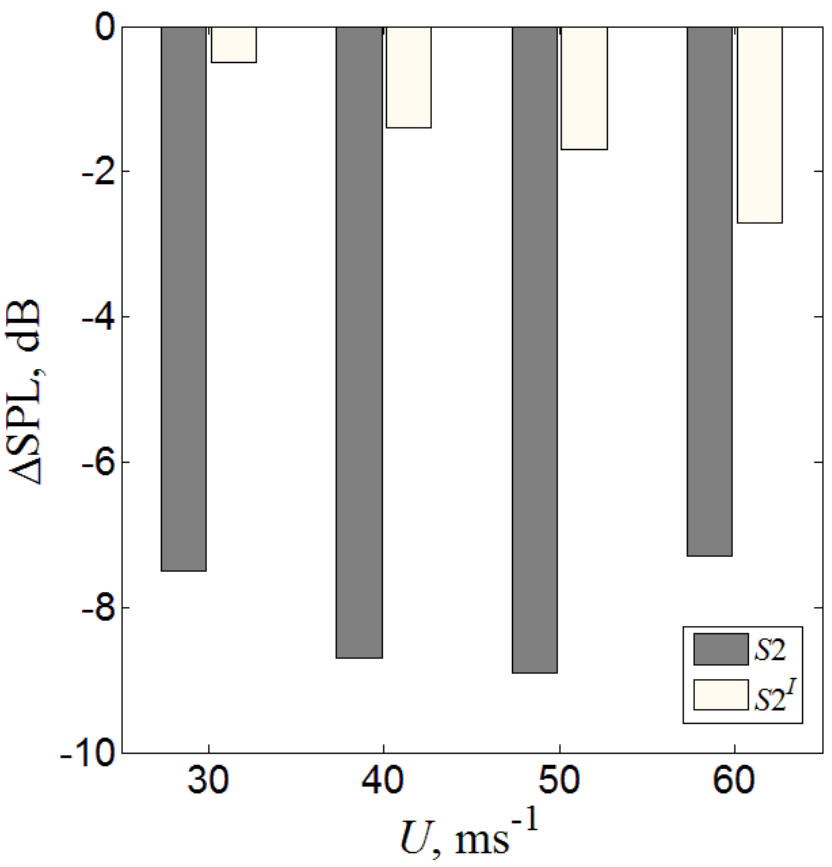

Fig. 5 (a) Distribution of Strouhal numbers $(f \varepsilon / U)$ that correspond to the narrowband tonal peak against flow velocity, and (b) Comparison of the $-\Delta$ SPL that corresponds to the tonal peal against flow velocity.

flow resistivity exists locally for the $S 2$ nonflat plate serrated trailing edge. Experimental evidence can be found in the flow visualisation images in Fig. 13 in ref $^{9}$. The $\Delta$ SPL associated with the tonal peak at $U=30,40,50$ and 60 $\mathrm{ms}^{-1}$ are shown in Fig. 5b.

When porous material is added to the sawtooth gaps of the nonflat plate serration, the local air flow resistivity increases and the trailing edge becomes a porous-serration. As shown in Fig. 4a-b, most of the poro-serrated trailing edges $\left(S 2^{I I}, S 2^{I I I}\right.$ and $S 2^{I V}$ ) cease to produce the narrowband tone noise in Zone II. This indicates that vortex shedding is now largely suppressed by the porous part of the poro-serrated trailing edge. This has been confirmed in the near wake hot wire measurements in the previous study in ${ }^{11}$.

The $S 2^{I}$ poro-serrated trailing edge, which has the lowest air flow resistivity at the sawtooth gaps $(\sigma)$, produces a small negative $\Delta$ SPL at $500 \leq f \leq 900 \mathrm{~Hz}$ encompassing both Zone I and Zone II. Comparing the acoustic spectra and $\Delta$ SPL between $U=20$ and $60 \mathrm{~ms}^{-1}$ in Fig. 6a, tone noise is still observed for the $S 2^{I}$ poro-serrated trailing edge. Using the same bluntness $\varepsilon=5.7 \mathrm{~mm}$, the resulting reduced frequency $(f \varepsilon / U)$ of the tone noise produced by the $S 2^{I}$ poro-serrated trailing edge, which averaged at 0.103 between $U=30$ and $60 \mathrm{~ms}^{-1}$, is shown in Fig. 5a. Although still demonstrating a Strouhal number dependency, this value is significantly lower than the 0.158 produced by the $S 2$ serrated trailing edge. From the $\Delta$ SPL plot in Fig. 6 b, moderate level of residual tone can still be radiated by the $S 2^{I}$ poro-serrated trailing edge at relatively high velocities. This is different with the $S 2$ case where strong level of tone noise can already be produced at low velocity, as shown in Fig. 5b. It shows that gradually increasing the $\sigma$ can reduce both the tone frequency and amplitude. Based on the results in Fig. 5a-b, and those already published in ${ }^{11}$, the minimum $\sigma$ occurs at around $8 \mathrm{kN} . \mathrm{s}^{-4} \mathrm{~m}^{-4}$. Below this value, low level of tonal noise is still radiated but the shedding frequency is shifted towards frequency Zone I. At $\sigma \geq 8 \mathrm{kN} . \mathrm{s}^{-4}$, the vortex shedding can be suppressed effectively and no noticeable tonal characteristic is found in the noise spectra across the velocities.

Back to Fig. 4b, another important feature achieved by the poro-serrated trailing edges in Zone II is their ability to significantly reduce the broadband noise, albeit there is also a large variation in the average level of noise reduction among the poro-serrated trailing edges. The $S 2^{I I}$ poro-serrated trailing edge produces the largest level of noise reduction, followed by the $S 2^{I I I}$ and then the $S 2^{I V}$. The peaks in $+\Delta$ SPL for these poro-serrated cases $\left(S 2^{I I}, S 2^{I I I}\right.$ and $S 2^{I V}$ ) all occur at $f=1.26 \mathrm{kHz}$, and the level of $+\Delta \mathrm{SPL}$ improves as $\sigma$ reduces. However, the $S 2^{I}$ poro-serrated trailing edge exhibits a slow recovery of $+\Delta \mathrm{SPL}$, which does not conform to the trend described above. This is likely 
(a)

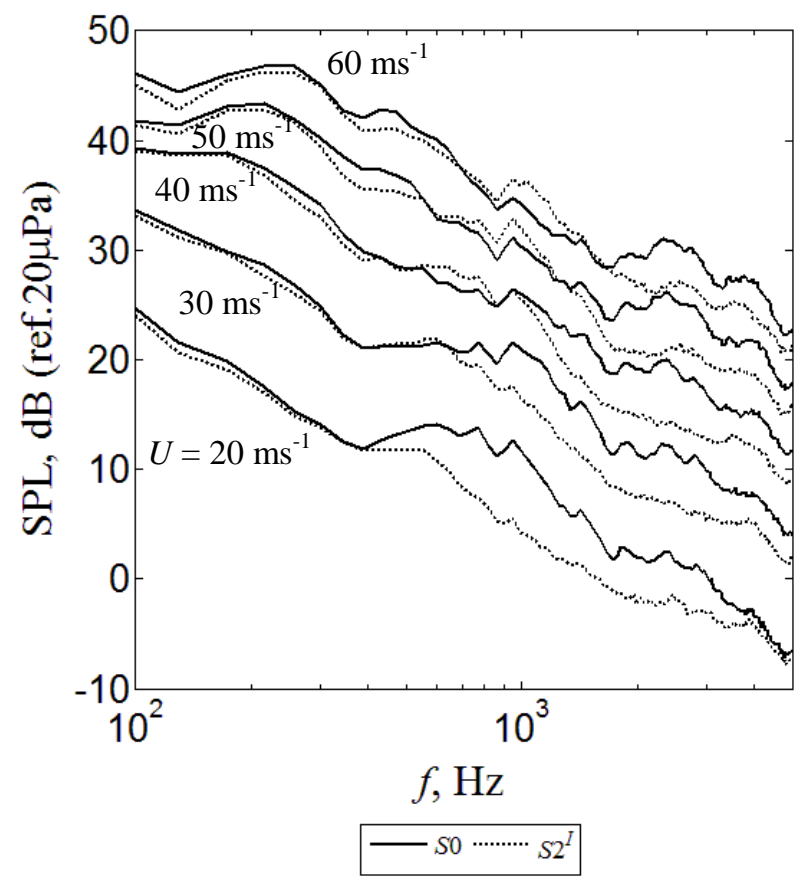

(b)

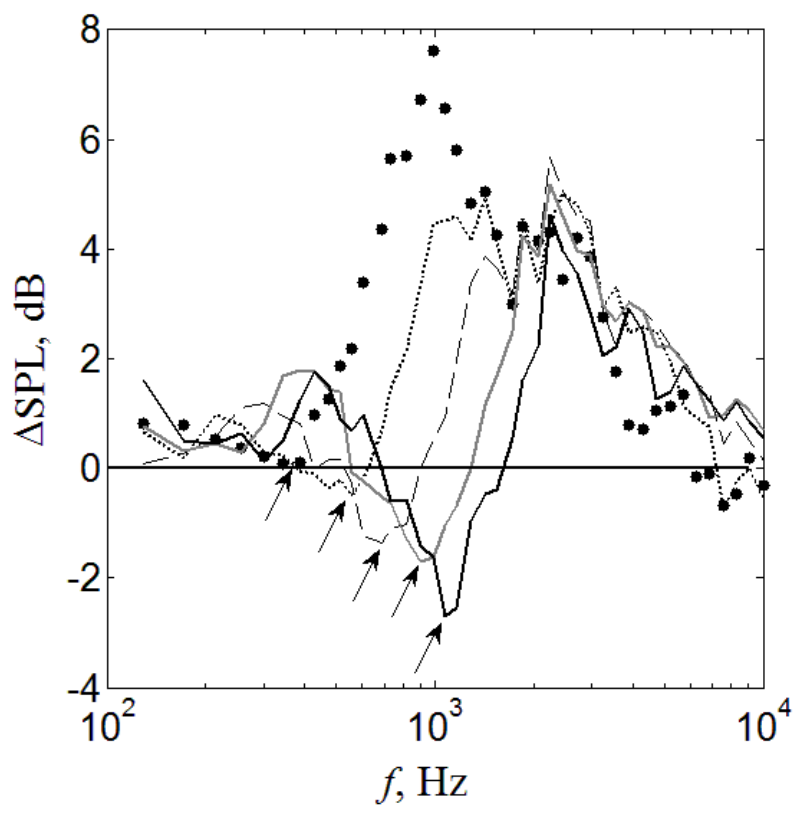

- $20 \mathrm{~ms}^{-1} \cdots \cdots \cdots \cdot 30 \mathrm{~ms}^{-1}--40 \mathrm{~ms}^{-1}-50 \mathrm{~ms}^{-1}-60 \mathrm{~ms}^{-1}$

Fig. 6 (a) Comparison of SPL at different $U$ between $S 0$ baseline and $S 2^{I}$ poro-serrated trailing edges, (b) $\triangle$ SPL of the $S 2^{I}$ poro-serrated trailing edge at different $U$.

to be caused by the frequency broadening of the tonal noise at the interface between Zone I and II. As a result, the corresponding peak in $+\Delta \mathrm{SPL}$ is shifted towards higher frequency at $f=1.45 \mathrm{kHz}$.

The poro-serrated trailing edges $\left(S 2^{I}, S 2^{I I}, S 2^{I I I}\right.$ and $\left.S 2^{I V}\right)$ all share the same serration parameters in $2 h, \varphi$ and $\lambda / h$, but they have different values of $\sigma$. Based on the above observation, the large variation in the level of broadband noise reduction among the poro-serrated trailing edges in this frequency zone seems to be predominantly caused by the effect of flow resistivity from the porous materials, where a change of local flow dynamic as a result can further interact with the serration sawtooth to cause a broadband noise reduction. This issue will be discussed later.

\section{Zone III}

As shown in Figs. 4a-b, the $S 2$ serrated, as well as the $S 2^{I}, S 2^{I I}, S 2^{I I I}$ and $S 2^{I V}$ poro-serrated trailing edges all demonstrate that they can achieve substantial broadband noise reduction in this large frequency zone. Focusing first on the $S 2$ serrated trailing edge in Fig. $4 \mathrm{~b},+\Delta$ SPL up to $6 \mathrm{~dB}$ can be achieved at this particular velocity. It is interesting to note that the vortex shedding, being described as an unwanted and adverse flow phenomenon in frequency Zone II, does not seem to be detrimental to the serration effect on the broadband noise reduction in frequency Zone III. In the context of nonflat plate serration, it has even been suggested that the presence of transverse flow within the sawtooth gaps is one of the pre-requisites for the manifestation of the serration effect ${ }^{14}$.

The first direct comparison between a conventional serrated trailing edge $(S 2)$ and poro-serrated trailing edge in this frequency zone confirms that the levels in $+\Delta \mathrm{SPL}$ achieved by the $S 2^{I}$ and $S 2^{I I}$ poro-serrated trailing edges are higher than the $S 2$ serrated trailing edge. For the $S 2^{I I I}$ poro-serrated trailing edge, when $\sigma$ increases to 41.2 kN.s.m ${ }^{-4}$, the level of broadband noise reduction in $+\Delta$ SPL starts to be lower than that achieved by the $S 2$ nonflat plate serrated trailing edge. This implies that the relatively high flow resistivity at the sawtooth gaps of the $S 2^{I I I}$ case begins to interfere negatively with the serration effect at this frequency zone. The $S 2^{I V}$ poro-serrated trailing edge, which has the largest $\sigma$ at the sawtooth gap, can only achieve a moderate $+\Delta$ SPL. It is indeed the lowest performer among all the trailing edge devices tested here. As the $\sigma$ increases, the porous material used in the sawtooth gaps becomes denser. This would render the poro-serrated trailing edge to gradually becoming a straight $S 0$ trailing edge again, thus undermining the serration effect and causing a drop in the performance of broadband noise reduction. 
In ascending order, the level of $+\Delta S P L$ achieved among the trailing edge devices is as follow: $S 2^{I V}, S 2^{I I I}, S 2, S 2^{I}$ and $S 2^{I I}$. Both the serrated $(S 2)$ and poro-serrated $\left(S 2^{I}, S 2^{I I}, S 2^{I I I}\right.$ and $\left.S 2^{I V}\right)$ trailing edges share the same serration parameters in $2 h, \varphi$ and $\lambda / h$, but they are different in $\sigma$. Therefore, whilst one of the mechanisms underpinning the broadband noise reduction is clearly by the serration effect, the flow resistivity at the sawtooth gaps represents another avenue to further improve the noise reduction capability in this frequency zone because the $S 2^{I}$ and $S 2^{I I}$ poro-serrated trailing edges have been shown to outperform the $S 2$ serrated trailing edge slightly.

\section{Zone IV}

This frequency zone marks the decline in the level of $+\Delta$ SPL achieved by both the serrated and poro-serrated trailing edge devices, where $\Delta \mathrm{SPL} \rightarrow 0$ as the frequency increases. Neither the sawtooth nor the porous material could exert significant impact on the acoustic spectrum at high frequency because self-noise radiated from the trailing edge by the dipole scattering of turbulent eddies is no longer the major noise source here.

(a)

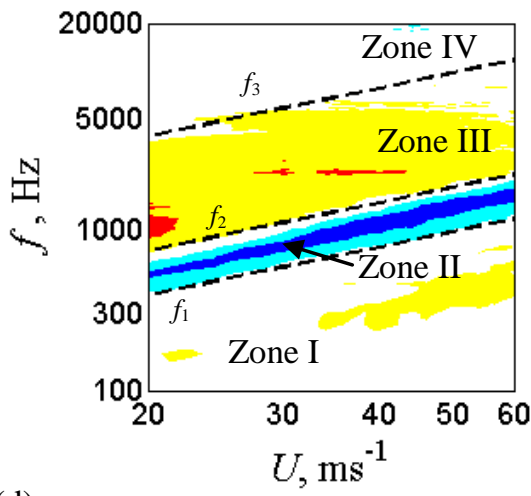

(d)

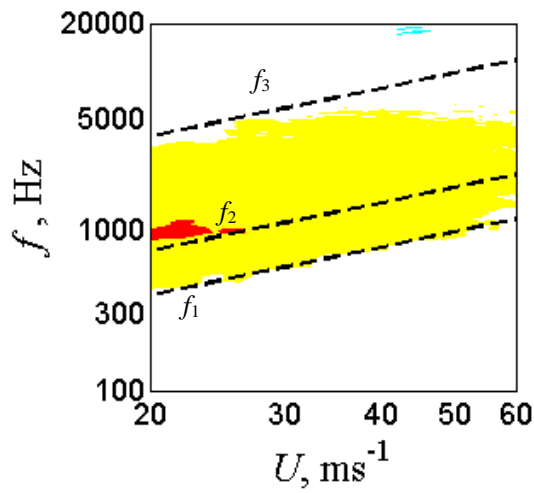

(b)

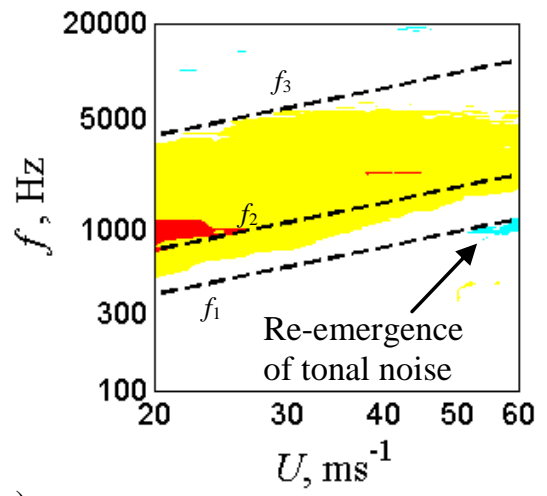

(e)

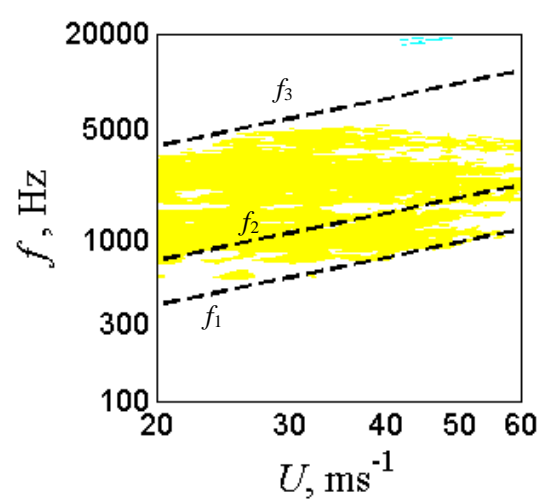

(c)

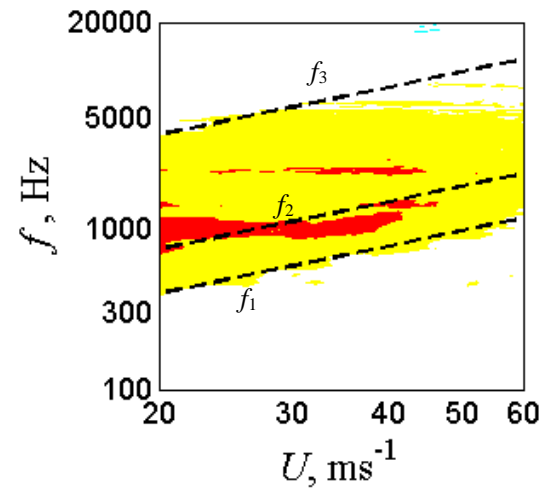

Noise

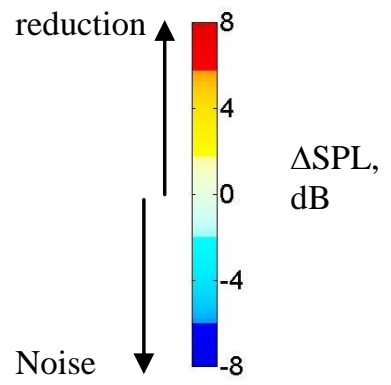

increase

Fig. 7 Contours of $\triangle \mathrm{SPL}$, dB produced by (a) $S 2$ serrated trailing edge, and (b) $S 2^{I}$, (c) $S 2^{I I}$, (d) $S 2^{I I I}$ and $S 2^{I V}$ poro-serrated trailing edges. The frequency Zones I, II, III and IV indentified in (a) are also applicable to (b)-(e).

\section{Noise Spectra at Other Velocities}

This section will examine the noise performance of the serrated and poro-serrated trailing edges at other velocities. Figures 7a-e show contour maps of $\triangle \mathrm{SPL}$ as a function of frequency and mean velocity $\left(U=20-60 \mathrm{~ms}^{-1}\right)$ for the $S 2, S 2^{I}, S 2^{I I}, S 2^{I I I}$ and $S 2^{I V}$, respectively. Note that the velocity resolution in these figures is $2 \mathrm{~ms}^{-1}$. The different frequency Zones I, II, III and IV identified earlier in Section IV are also reproduced here as a function of the velocity, which are defined by: 


$$
\begin{gathered}
f_{1}(U)=20 . U^{1.0} \\
f_{2}(U)=38 . U^{1.0} \\
f_{3}(U)=20 . U^{1.0}+5500
\end{gathered}
$$

Both $f_{1}$ and $f_{2}$ represent the lower and upper limits of the narrowband tone noise in Zone II produced by the $S 2$ serrated trailing edge. They are both proportional to $U^{1.0}$, indicating a Strouhal number relationship. Assuming a characteristic length scale equal to the sawtooth bluntness $\varepsilon$, the corresponding Strouhal numbers are 0.11 and 0.22, respectively. As indicated in Figs. 7a-e, the variation of $f_{3}$ with $U$ is kept at the same velocity scaling that produces an interface that could still reasonably separate frequency Zones III and IV. The variation of $f_{3}$ as a function of $U$ in Eq. (2), which is fitted against the $S 2^{I I}$ poro-serrated trailing edge, is essentially an offset of $f_{1}$ with the same gradient. Therefore the frequency difference $\left(f_{3}-f_{1}\right)$ will be the same across the $U$. Note that the same definition of $f_{3}$ also applies to other trailing edge devices. The reason for choosing the $S 2^{I I}$ poro-serrated trailing edge as the representative one is because it produces the largest frequency bandwidth of $+\Delta$ SPL among others. This characteristic is more desirable for the NPM analysis, which will be described later.

In the previous section, the $S 2$ serrated trailing edge at $U=40 \mathrm{~ms}^{-1}$ indicates that noise reduction can be achieved in the frequency Zone I. As shown in Fig. 7a, noise reduction in Zone I also occurs across a large velocity range especially towards the high velocity end. Significant broadband noise reduction can be found in the frequency Zone III. However, high level of noise increase caused by the vortex shedding tone dominates the frequency Zone II.

For all the poro-serrated trailing edges in Figs. 7b-e, no apparent noise reduction, nor increase, can be observed in the frequency Zone I. Nevertheless, substantial improvement in the overall noise control performance is demonstrated by the: (1) total suppression of tonal rungs in Zone II, and (2) significant broadband noise reduction $(\triangle \mathrm{SPL}>8 \mathrm{~dB})$ in Zones II and III.

A qualitative examination of Figs. 7b-e can easily establish that the level and frequency bandwidth of the broadband noise reduction is influenced by the flow resistivity at the sawtooth gaps $(\sigma)$ of the poro-serrated trailing edges. In order to quantify the difference, an overall Noise Performance Metric (NPM) is defined:
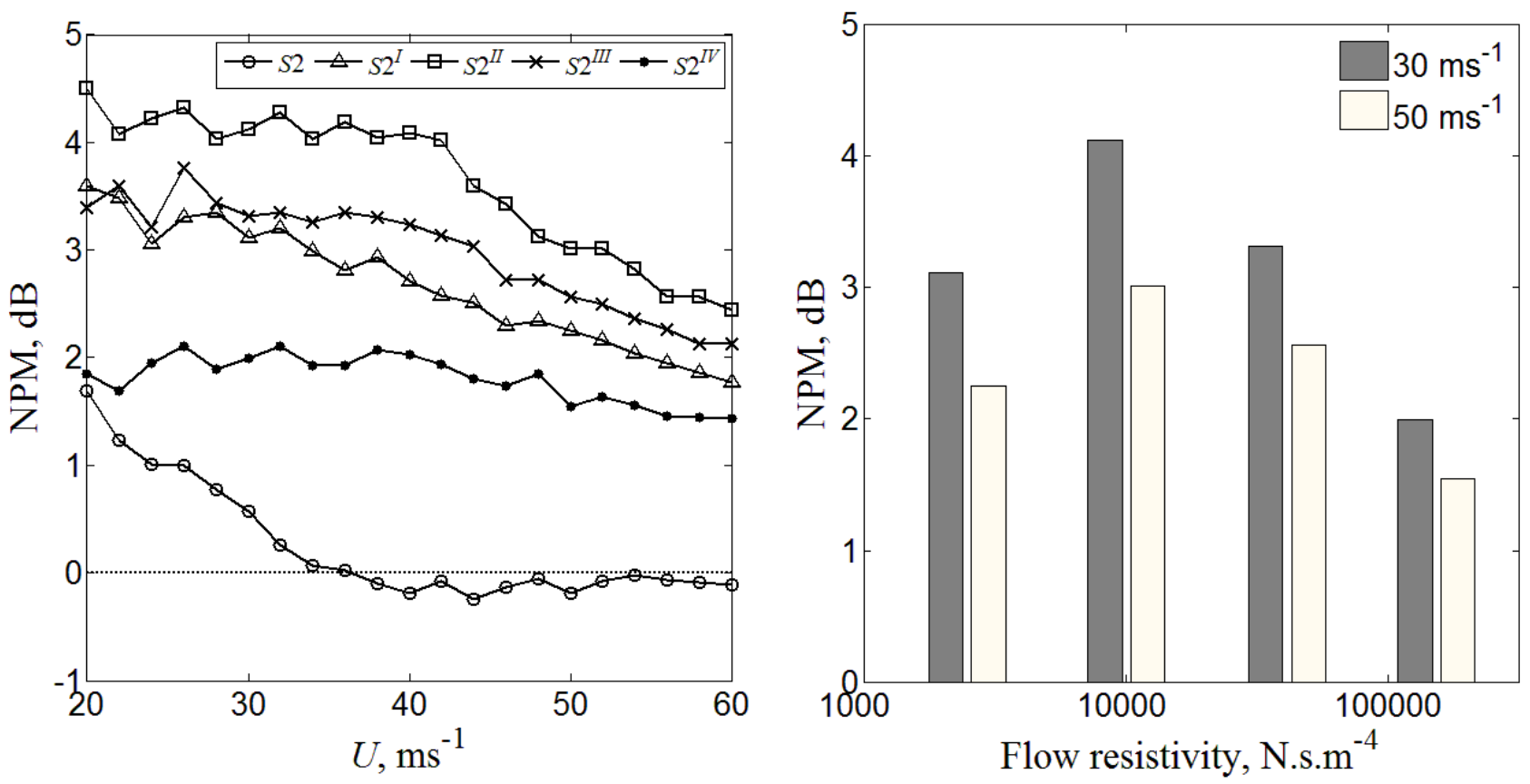

Fig. 8 Distributions of NPM, dB for frequency Zones (II + III), or $J=\left(f_{1}, f_{3}\right)$ against (a) $U$, and (b) flow resistivity at the sawtooth gaps. 


$$
\mathrm{NPM}=10 \log _{10}\left(\int_{f \in J} p^{\prime^{2}} d f / \int_{f \in J} p_{\text {Serration }}^{\prime^{2}} d f\right),
$$

where $J$ is a finite frequency bandwidth that could vary with velocity, and $p^{\prime}$ is the pressure fluctuation measured by the microphone. This makes the NPM sensitive to the choice of $J$ but nevertheless it is still a useful measure of the noise performance achieved by the serrated trailing edge at a particular $U$.

Figure 8a presents the variations of NPM against $U$ for $S 2, S 2^{I}, S 2^{I I}, S 2^{I I I}$ and $S 2^{I V}$ when $J=\left(f_{1}, f_{3}\right)$, which represents the combination of frequency Zones II and III. It can be seen that most of the NPM for the $S 2$ serrated trailing edge is low and even becoming negative at $U>36 \mathrm{~ms}^{-1}$. This is largely due to the presence of tonal rung in the noise spectrum. Next, the NPM fluctuates between 2 and $3.5 \mathrm{~dB}$ for the $S 2^{I}$ poro-serrated trailing edge over the velocity range investigated here. Despite the rather low level of $\sigma$ in the $S 2^{I}$ case, the result indicates that: (1) the tonal rung has already been weakened, and (2) the overall noise performance is largely characterised by the reduction in broadband noise. For the $S 2^{I I}$ poro-serrated trailing edge with a larger $\sigma$, the average level of NPM improves to between 2.5 and $4.5 \mathrm{~dB}$. However, a further increase of $\sigma$ to the level corresponding to the $S 2^{I I I}$ does not result in further improvement of the NPM. Instead, the performance is just slightly better than the $S 2^{I}$ case. Finally, for the largest $\sigma$ level tested in this study, the $S 2^{I V}$ poro-serrated trailing edge actually achieves the lowest overall performance in NPM between 1.5 and $2 \mathrm{~dB}$. This is caused by the use of dense porous material that renders the trailing edge to becoming more solid across the span, thus weakening the serration effect. The results in Fig. 8a also show that NPM produced by most of the poro-serrated trailing edges (except the $S 2^{I V}$ type) reduces as $U$ increases. Figure $8 \mathrm{~b}$ plots the variation of NPM against $\sigma$ when $J=\left(f_{1}, f_{3}\right)$ for two velocities. It can be seen that the optimum value of $\sigma$ is about $10 \mathrm{kN} . \mathrm{s} . \mathrm{m}^{-4}$.

(a)

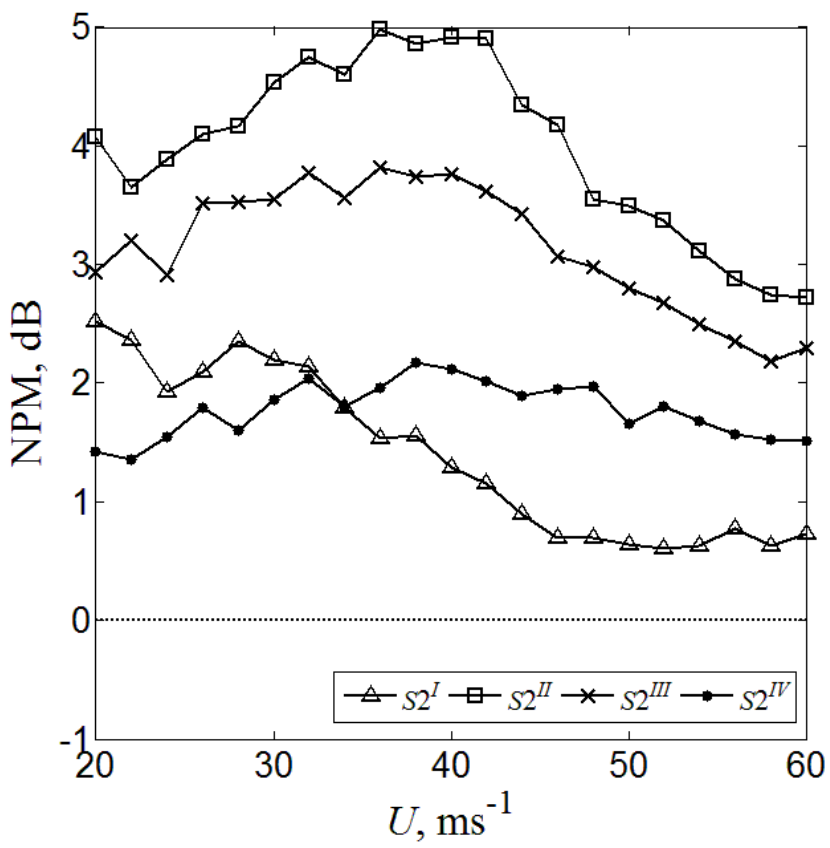

(b)

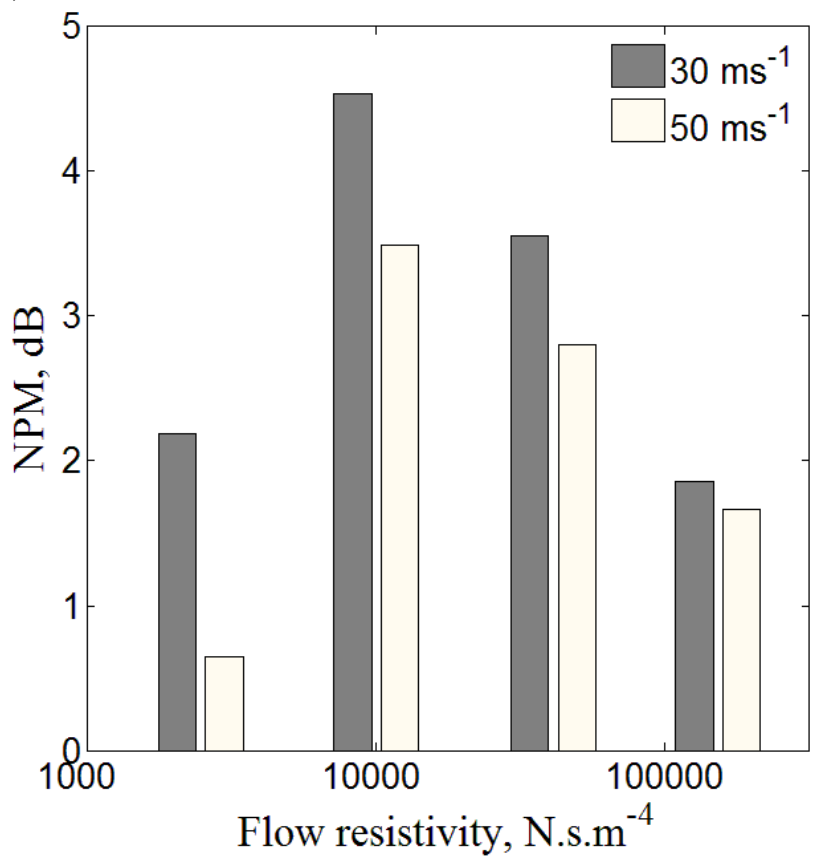

Fig. 9 Distributions of NPM, dB for frequency Zone II only, or $J=\left(f_{1}, f_{2}\right)$ against (a) $U$, and (b) flow resistivity at the sawtooth gaps.

The NPM presented above represents a large frequency bandwidth that combines Zones II and III together. The examination will now focus on the NPM of Zone II and Zone III, individually. Figure 9a presents the variations of NPM against $U$ for $S 2^{I}, S 2^{I I}, S 2^{I I I}$ and $S 2^{I V}$ when $J=\left(f_{1}, f_{2}\right)$, i.e. the frequency Zones II. Note that the NPM for the $S 2$ serrated trailing edge is not included here because of the expected domination by the vortex shedding tonal noise. The most notable feature in Fig. 9a is that large variation of NPM is produced among the poro-serrated trailing 
edges. This indicates that the NPM in this frequency zone is more sensitive to the level of $\sigma$. In ascending order, the level of NPM achieved among the poro-serrated trailing edges is as follow: $S 2^{I V}, S 2^{I I I}$ and $S 2^{I I}$. For the $S 2^{I}$ poroserrated trailing edge, however, its NPM is almost in the same category as the $S 2^{I V}$ counterpart at $U \leq 34 \mathrm{~ms}^{-1}$, but undergoes a large drop in NPM as $U$ increases up to $46 \mathrm{~ms}^{-1}$. At $U>46 \mathrm{~ms}^{-1}$, the NPM does not change much but remains low. The reason that causes the drop in noise performance for the $S 2^{I}$ poro-serrated trailing edge at high velocity is due to the re-emergence of a weak vortex shedding at the sawtooth gaps (see Fig. 7b). Figure 9b plots the variation of NPM against the $\sigma$ when $J=\left(f_{1}, f_{2}\right)$. Similarly, the optimum value of $\sigma$ is also at $10 \mathrm{kN} . s . \mathrm{m}^{-4}$.

(a)

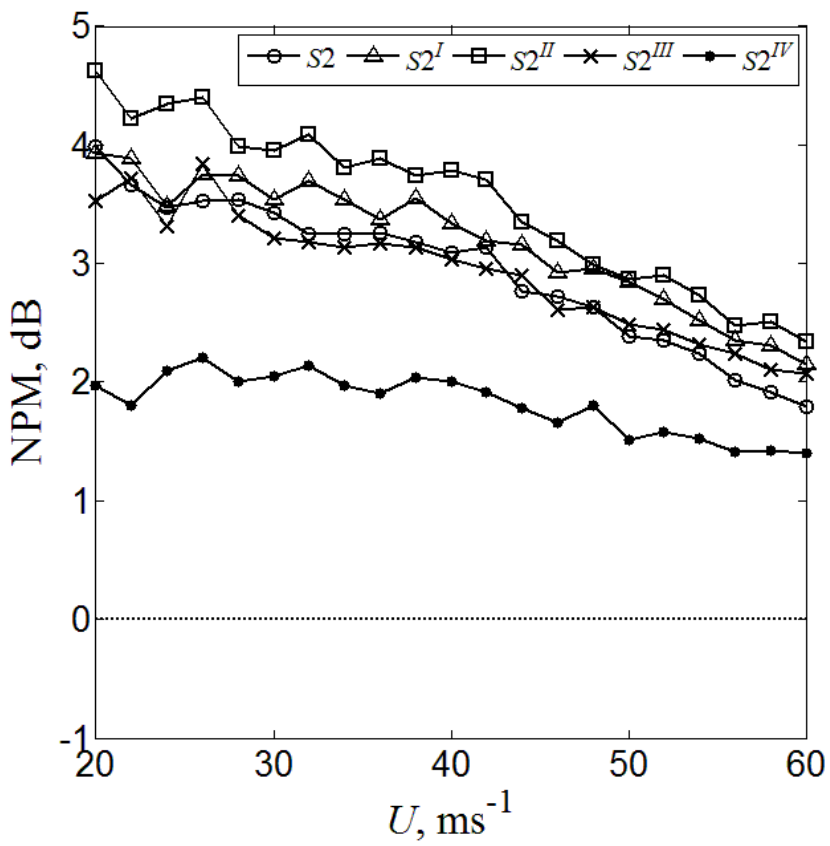

(b)

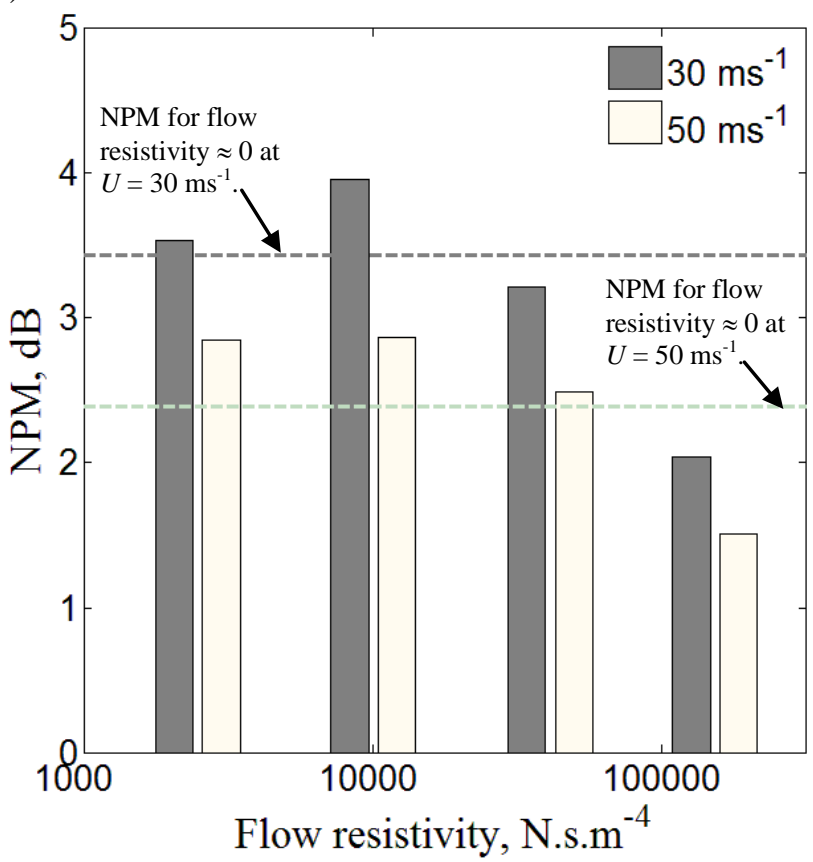

Fig. 10 Distributions of NPM, dB for frequency Zone III only, or $J=\left(f_{2}, f_{3}\right)$ against (a) $U$, and (b) flow resistivity at the sawtooth gaps.

Based on the results presented thus far, choosing the right porous material at the sawtooth gaps seems to be able to improve the broadband noise reduction. However, question remains of whether an optimised poro-serrated trailing edge could perform better than the $S 2$ serrated trailing edge where $\sigma=0$. To provide some hints, this time the NPM in frequency Zone III only, i.e. $J=\left(f_{2}, f_{3}\right)$, will be examined. The corresponding NPM against $U$ for $S 2$, $S 2^{I}, S 2^{I I}, S 2^{I I I}$ and $S 2^{I V}$ is shown in Fig. 10a. For the $S 2$ serrated trailing edge, where the influence of tonal rung no longer exists at this frequency zone, the corresponding NPM improves significantly and it has achieved between 2 and $4 \mathrm{~dB}$ broadband noise reduction across the whole velocity range investigated here. The average level of NPM achieved by the $S 2$ serrated trailing edge is actually quite similar to the $S 2^{I I I}$ poro-serrated trailing edge, where the level of $\sigma$ is the second highest. However, the average levels of NPM achieved by the $S 2^{I}$ and $S 2^{I I}$ poro-serrated trailing edges still outperform the $S 2$ serrated trailing edge. Finally, the NPM for the $S 2^{I V}$ poro-serrated trailing edge continue to remain the lowest. Figure $10 \mathrm{~b}$ plots the variation of NPM against the $\sigma$ when $J=\left(f_{2}, f_{3}\right)$. In this time the NPM for the $S 2$ serrated trailing edge, where $\sigma=0$, is indicated in the figure. Between $2.5 \leq \sigma \leq 10 \mathrm{kN} . \mathrm{s} . \mathrm{m}^{-4}$, the results in Fig. 10b clearly demonstrate that the poro-serrated trailing edge can achieve slightly higher level of broadband noise reduction (NPM $\sim 0.5 \mathrm{~dB}$ ) than the $S 2$ serrated trailing edge.

Generally speaking, the most optimum level of $\sigma$ is about $10 \mathrm{kN} . \mathrm{s} . \mathrm{m}^{-4}$. The performance in NPM then deteriorates as $\sigma>10 \mathrm{kN} . s . \mathrm{m}^{-4}$. When $\sigma \rightarrow \infty$, the poro-serrated trailing edge will return to the $S 0$ baseline trailing edge where the NPM $\rightarrow 0$.

An interesting aspect when $\sigma<10 \mathrm{kN} . s . \mathrm{m}^{-4}$, for example the $S 2^{I}$ case at $2.5 \mathrm{kN} . \mathrm{s} . \mathrm{m}^{-4}$, is that it has a potential to outperform the $S 2^{I I I}$ counterpart $\left(\sigma=41.2 \mathrm{kN} . \mathrm{s} . \mathrm{m}^{-4}\right)$ in terms of the level of broadband noise reduction (see Fig. 10b). However, the $S 2^{I}$ poro-serrated trailing edge can also be undermined by the re-emergence of weak vortex 
shedding at the sawtooth gaps when $\sigma$ is too low (see Figs. 7b and 9b). As a result, when a larger frequency bandwidth is considered, the overall noise performance of the $S 2^{I}$ poro-serrated trailing edge is not as good as the $S 2^{I I I}$ counterpart (see Fig. 8b).

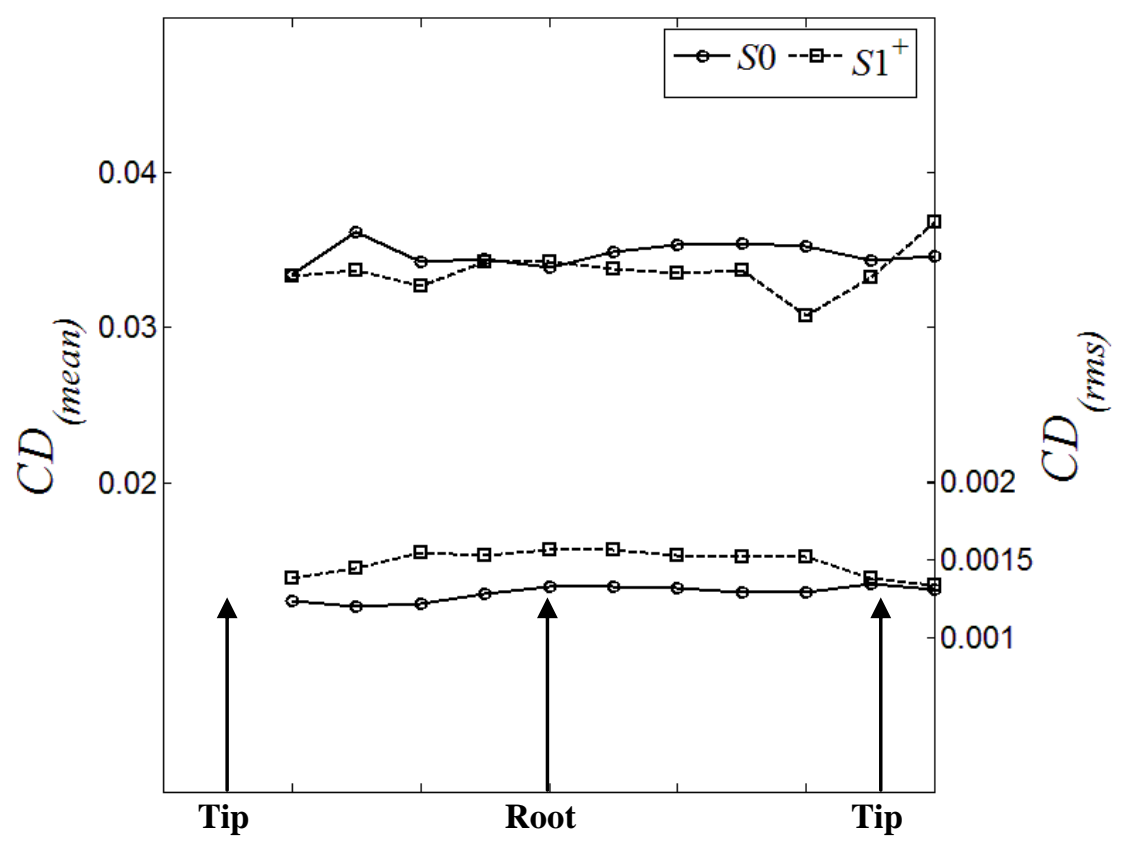

Fig. 11 Distributions of $C_{D(m e a n)}$ and $C_{D(r m s)}$ across a unit wavelength of the sawtooth in the spanwise $(z)$ direction at $x / C=$ 1.03.

\section{Very Near Wake Produced by the Poro-Serrated Trailing Edge}

This section discusses the results of some of the velocity measurements at the very near wake region for the same type of aerofoil with baseline straight, poro-serrated and nonflat plate serrated trailing edges. The poro-serrated trailing edge used in the wake flow study, denoted here as $S 1^{+}$, has a narrower serration angle, but also the same serration amplitude compared to the $S 2^{I}-S 2^{I V}$ cases presented previously (See Table 1). The porous material used to fill the sawtooth gap is the nickel-chromium foam where the flow resistivity, $\sigma \approx 8.0 \mathrm{kN} . \mathrm{s} . \mathrm{m}^{-4}$. A nonflat plate type serrated trailing edge, $S 1$, which has exactly the same serration parameters as $S 1^{+}$, but with $\sigma$ at the sawtooth gap equal to 0 , is also investigated. Noise performance for these two types of trailing edge devices has already been reported elsewhere ${ }^{11}$, where up to $7 \mathrm{~dB}$ broadband noise reduction can be achieved. As explained in Section II.A, the unsteady velocity was measured by a single hot wire probe traversing in a $y-z$ plane. The streamwise distance of the measurement plane was conducted at $x / C=1.03$, i.e. about $5 \mathrm{~mm}$ from the trailing edge. This distance is usually considered as the very near wake where the viscous sublayer is still well separated from the neighbouring inertial sublayer, and the velocity deficit is large ${ }^{18}$. The incoming jet velocity was set at $40 \mathrm{~ms}^{-1}$, and the angle of attack was also at zero degree. For the corresponding noise spectra showing the broadband noise reduction achieved under these conditions by the $S 1$ and $S 1^{+}$, the readers can refer to Fig. 5a in ref ${ }^{11}$.

The first aerodynamic parameter to be discussed here is the drag coefficient, $C_{D}$ produced by the $S 0$ and $S 1^{+}$ trailing edges (the result for the $S 1$ case is omitted here for brevity). Wake velocity profiles of the mean $(u)$ and fluctuating $\left(u_{r m s}\right)$ components were used to calculate the mean and fluctuating component of $C_{D}$, given by $C_{D}=$ $C_{D(\text { mean })}+C_{D(\text { rms) }}$. More specifically, for a unit span of the aerofoil:

$$
C_{D}=\frac{2}{C} \int_{-\infty}^{\infty} \frac{u}{u_{\infty}}\left(1-\frac{u}{u_{\infty}}\right) d y+\frac{2}{C} \int_{-\infty}^{\infty}\left(\frac{u_{r m s}}{u_{\infty}}\right)^{2} d y,
$$


where $u_{\infty}$ is the mean velocity value in the potential flow region, thus outside the shear layer. In this study, a total of 21 wake profiles across a spanwise distance of $10 \mathrm{~mm}$ were measured. Therefore the spanwise spatial resolution, $\Delta z$ $=0.5 \mathrm{~mm}$. Each wake profile contains 39 measurement points across a vertical distance of $28 \mathrm{~mm}$. The vertical spatial resolution, $\Delta y$, is arranged as the following: $\Delta y=1 \mathrm{~mm}$ at $14 \leq y \leq 5 \mathrm{~mm}$, and $\Delta y=0.5 \mathrm{~mm}$ at $5 \leq y \leq 0 \mathrm{~mm}$. Here, $y=0 \mathrm{~mm}$ denotes the tip of the trailing edge or centreline of the wake profile, which is also the location where the maximum velocity deficit occurs. Therefore, all positive $y$ represents the upper half of the aerofoil, whilst the lower half of the aerofoil mirrors exactly the above grids, giving all negative $y$.

Figure 11 shows the distribution of $C_{D(\text { mean) }}$ and $C_{D(\mathrm{rms})}$ across approximately a unit wavelength of the sawtooth. The $C_{D(\text { mean) }}$ produced by the baseline $S 0$ trailing edge is constant at about $0.035 \pm 0.0008$, with a slight fluctuation of data across the $z$. The $C_{D \text { (mean) }}$ produced by the poro-serrated trailing edge, $S 1^{+}$, averaged at $0.034 \pm 0.0014$. On the other hand, the poro-serrated trailing edge produces $19 \%$ larger $C_{D(\mathrm{rms})}$ than the baseline trailing edge. It should be noted that the $C_{D(\mathrm{rms})}$ produced by both trailing edges are order of magnitude lower than the $C_{D \text { (mean) }}$ counterpart. Therefore the main contributor to the total drag comes from the $C_{D \text { (mean). }}$

It should also be noted that the values of the $C_{D(\text { mean })}$ may seem generally high at first sight, but this is because the boundary layers near the leading edge of the aerofoil had been artificially tripped into turbulent on both sides. Therefore the values are associated with a turbulent boundary layer generated on an aerofoil.
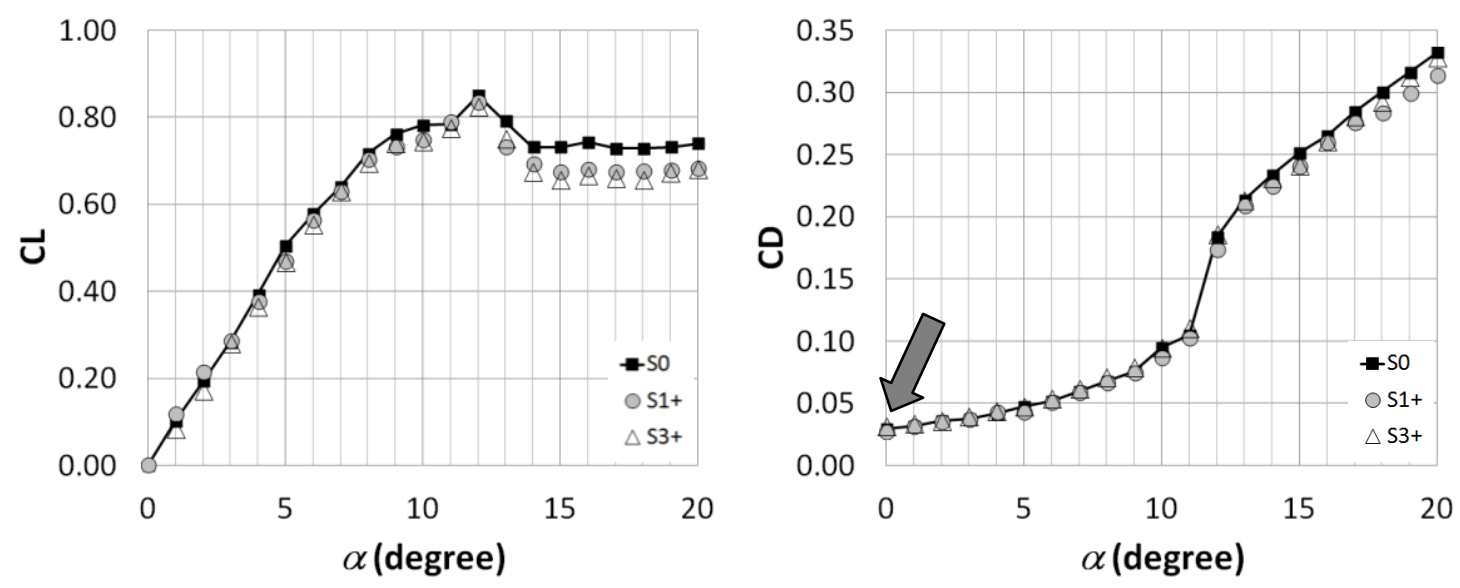

a)

b)

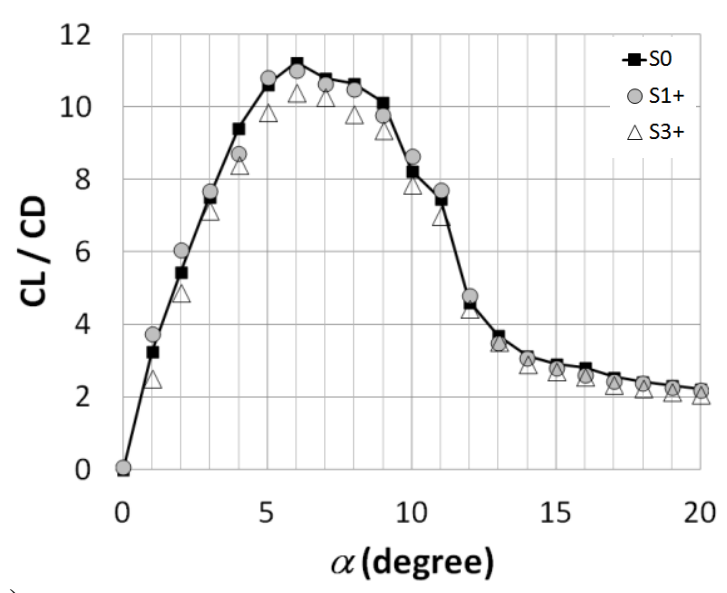

c)

Fig. 12 Comparisons of the aerodynamic forces produced by the $S 0$ trailing edge (baseline), and $S 1^{+}$and $S 3^{+}$poro-serrated trailing edges at $U=30 \mathrm{~ms}^{-1}$ for a) CL; b) $\mathrm{CD}$ and c) CL/CD against angles of attack, $\alpha$. All the data was obtained by a force balance. All figures are reproduced from ref ${ }^{11}$. 
Before further discussion of the results in Fig. 11, it would be necessary to establish the accuracy of the drag coefficient determined from the very near wake velocity profile. This is a classical method based on integration of the velocity deficit across the wake, but an assumption might need to be made a priori where an ambient pressure should already be established at the measurement points, i.e. sufficiently downstream of the aerofoil body. Now, the momentum thickness of the wake, $\theta$, can be defined as:

$$
\theta=\int_{-\infty}^{\infty} \frac{u}{u_{\infty}}\left(1-\frac{u}{u_{\infty}}\right) d y .
$$

Therefore,

$$
C_{D(\text { mean })}=\frac{2}{C} \theta \text {. }
$$

Hah and Lakshiminarayana ${ }^{18}$ studied the near wake of a NACA0012 aerofoil (same as the current one used here) at various angles of attack. Using the same definition as Eq. (5), they have shown that the predicted and measured $\theta$ are constant at $1.0 \leq x / C \leq 2.5$ (see Fig. 9 in $\mathrm{ref}^{18}$ ). The measurement location of $x / C=1.03$ in the current study is therefore likely to yield the same $C_{D(\text { mean) }}$ value as those obtained in the far wake. To prove this point further, a direct drag measurement was performed in a separate wind tunnel (with a closed-working section) using a force balance. More detail of the experimental setup can be found in $\mathrm{ref}^{11}$. The measured drag coefficient is re-produced here in Fig. 12, where the $C_{D}$ at zero degree angle of attack are 0.030 and 0.028 for the $S 0$ and $S 1^{+}$trailing edges, respectively. These values obtained by the direct force measurement method are indeed similar to the $C_{D(\text { mean })}$ obtained by the wake velocity profile technique discussed previously.

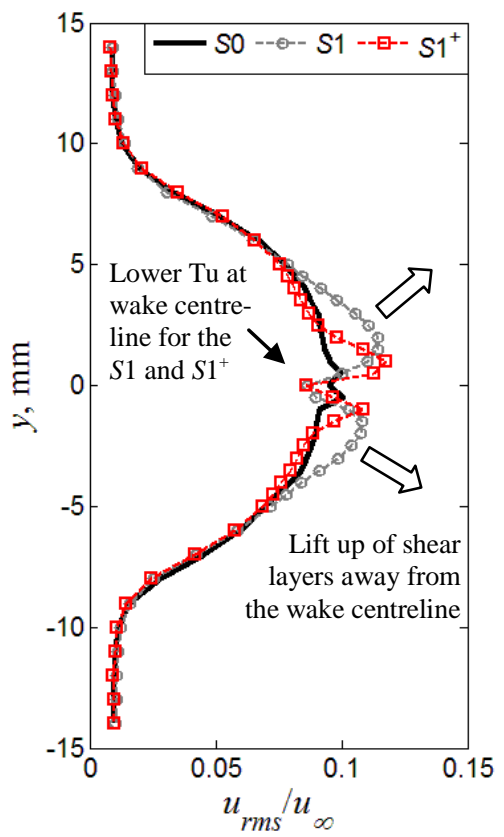

Tip

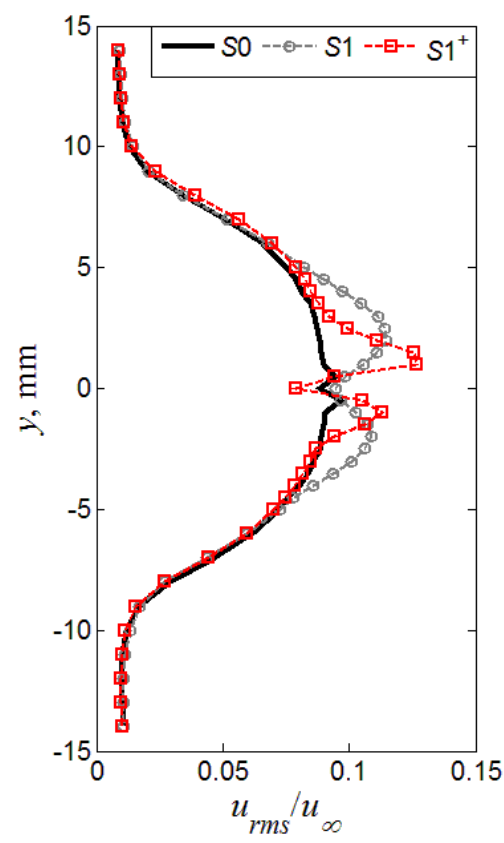

Mid-point

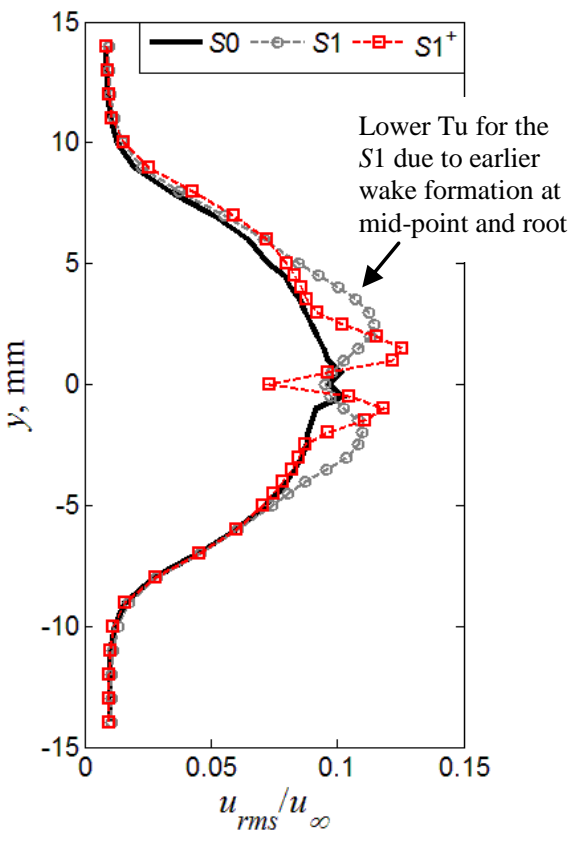

Root

\section{Fig. 13 Very near wake $(x / C=1.03)$ turbulent velocity profiles at the sawtooth tip, mid-point and sawtooth root.}

To summarise, adding porous foams to the sawtooth gaps of the poro-serrated trailing edge will not increase the overall drag coefficient at low blade loading or angle of attack. In addition, the current results suggest that a poroserrated trailing edge can even produce a slightly lower $C_{D(\text { mean })}$ than the baseline straight trailing edge. It does increase the $C_{D(\mathrm{rms})}$, but this will have little effect on the overall drag force. It is interesting to find that the values of $C_{D(\mathrm{rms})}$ as a function of $z$ varies according to the sawtooth geometry. From Fig. $11, C_{D(\mathrm{rms})}$ produced by the $S 1^{+}$ attains a maximum at region between the sawtooth root and the inner part of the oblique edges. When it is approaching the sawtooth tip, the $C_{D(\mathrm{rms})}$ produced by the $S 1^{+}$will revert back almost to the same value as the $S 0$ 
counterpart. Because the $C_{D(\mathrm{rms})}$ is essentially the integration of the square of the turbulence intensity across the wake, the result suggests that adding porous foams to the sawtooth gaps of the poro-serrated trailing edge will increase the turbulence characteristics locally.

This prompts a question of which part of the wake structure actually exhibits a higher turbulence characteristic when a poro-serrated trailing edge $S 1^{+}$, or a nonflat plate serrated trailing edge $S 1$, is used. Figure 13 shows three fluctuating velocity profiles at locations that coincide the sawtooth tip, mid-point of the oblique edge (referred herein as "mid-point") and root, respectively. The results demonstrate that the two shear layers produced by the poro-serrated trailing edge $S 1^{+}$have a higher turbulence intensity level than those produced by the baseline straight trailing edge, $S 0$. The shear layers in the wake produced by the $S 1^{+}$trailing edge, which are mostly originated from the upstream buffer layer, also lifted up from the wall. The relatively low flow resistivity of the porous medium $(\sigma=$ $8.0 \mathrm{kN} . \mathrm{s} . \mathrm{m}^{-4}$ ) near the trailing edge should encourage flow "communication" between the upper and lower surfaces, thereby reducing the unsteady $\Delta P$. Therefore it is reasonable to conjecture that the lifting up of these shear layers is a reaction to the opposing unsteady forces from underneath, while the intensified turbulence characteristic of the shear layer is caused by the enhanced turbulent flow mixing, with a possible contribution from the increased surface roughness. Interestingly, this flow behaviour can also be found in the region close to the sawtooth tip. This indicates that a certain level of cross flow exists along the aerofoil span near the trailing edge ${ }^{14}$.

The lifting up of the shear layers is even more pronounced for the nonflat plate serrated trailing edge, $S 1$. Because of the virtually zero flow resistance and the resulting large scale vortex shedding at the sawtooth gaps, the shear layers across the whole span are universally thicker and further away from the wake centreline than the shear layers produced by the poro-serrated trailing edge $S 1^{+}$. The level of turbulence intensity of the shear layers produced by the $S 1$ serrated trailing edge is similar to those produced by the $S 1^{+}$counterpart at the sawtooth tip, but lower at the mid-point and sawtooth root regions. This could be due to the fact that the wake starts to form at more upstream $(x / C<1.0)$ at the mid-point and sawtooth root, hence by the time it reaches the measurement point at $x / C=1.03$, the turbulence level has already decayed considerably. For the shear layers developed in the $S 1$ case, and in a direction from the sawtooth root to the sawtooth tip, their maxima in the turbulence intensities decrease in height $(y)$ almost linearly. It is important to note that the sawtooth tip has exactly the same aerofoil cross section as the baseline straight trailing edge. Without external influences, the boundary layer and wake developments for these two cases should be the same. However, at the sawtooth tip of the $S 1$ and $S 1^{+}$cases, the shear layers are found to develop further away from the wake centreline than the shear layers produced by the baseline straight trailing edge $S 0$. This indicates the presence of flow mixing obliquely in a direction from the sawtooth root to the sawtooth tip as soon as the wake is first formed at the root.

One important consequence of promoting the lift up of the intensified shear layers near the $\mathrm{S1}^{+}$poro-serrated trailing edge, as well as the $S 1$ nonflat plate serrated trailing edge, is the reduction of turbulence intensity in the wake centreline. This is manifested in the turbulent velocity profiles in Fig. 13, where the turbulence intensities at $y$ $\approx 0$ pertaining to the sawtooth tip, mid-point and root regions all exhibit lower level of turbulence intensity than the $S 0$ straight trailing edge. It is reasonable to assume that the effectiveness of turbulent broadband noise scattering at the sawtooth tip, oblique edge and sawtooth root is related to the level of turbulence at the near wall region. The turbulence characteristics at the very near wake, which still retains most of the original characteristics of the upstream boundary layer, offer an avenue for the study of noise reduction mechanism by the serration. Previously, it has already been observed that the turbulent velocity profiles at the very near wake pertaining to the sawtooth tip for both the $S 1$ and $S 1^{+}$trailing edge devices are markedly different to that produced by a $S 0$ straight trailing edge, although geometrically the edge (or tip) is the same at this particular $z$ location. This indicates that the mechanism of turbulent broadband noise reduction by serration is related to the dynamic of flow in the sawtooth gap and the way it interacts with the boundary layer at the sawtooth tip.

\section{Discussion}

This paper reports an experimental study on the aeroacoustic properties of a NACA0012 aerofoil with a nonflat plate type serrated trailing edge ( $S 2)$, and a number of poro-serrated trailing edge devices $\left(S 2^{I}, S 2^{I I}, S 2^{I I I}\right.$ and $\left.S 2^{I V}\right)$ that contain porous materials of different air flow resistivity at the sawtooth gaps. Both the nonflat plate serrated and poro-serrated trailing edges in the " $S 2$ " family share the same serration angle and sawtooth length. Investigation of the very near wake properties of the aerofoil was also performed. There are two trailing edge devices used in the flow measurement. These are the nonflat plate serrated trailing edge $(S 1)$ and a poro-serrated trailing edge $\left(\mathrm{S1}^{+}\right)$. The "S1" family also has the same serration angle and serration length (see Table 1). All these trailing edge devices, 
when integrated to an aerofoil body, will retain the original aerofoil shape. The free field noise measurements were carried out inside an aeroacoustic wind tunnel facility.

The nonflat plate type serrated trailing edge $(S 2)$, which has no flow resistivity at the sawtooth gaps $(\sigma=0)$, is shown to be capable of achieving reduction in the turbulent broadband noise. However, very large level of narrowband tone noise (Strouhal number $\approx 0.158$ ) is also radiated due to the strong vortex shedding on the sawtooth gaps. This tone noise significantly undermines the overall noise performance of the nonflat plate serrated trailing edge.

Other results demonstrate that having a low flow resistivity $\left(\leq 2.5 \mathrm{kN} . \mathrm{s} . \mathrm{m}^{-4}\right)$ at the sawtooth gaps in the poroserrated trailing edge configuration can reduce the noise level of the bluntness-induced vortex shedding tone at low frequency, but it cannot lead to a complete suppression. The averaged-Strouhal number associated with this residual narrowband tone is 0.103 across the flow velocity range. This value is much lower than the one produced by the nonflat plate serrated trailing edge. Although this particular poro-serrated trailing edge has demonstrated the potential to outperform the nonflat plate serrated trailing edge in terms of broadband noise reduction at high frequency, its overall noise performance is not the best because the residual vortex shedding tone is still present in the noise spectra.

For very high flow resistivity $\left(\geq 153 \mathrm{kN} . \mathrm{s} . \mathrm{m}^{-4}\right)$ at the sawtooth gaps, the rather dense porous material will almost revert the poro-serrated trailing edge back to a baseline, straight trailing edge. As a result, the effect of serration on the broadband noise radiation is gradually diminishing, which can lead to a reduced level of broadband noise reduction. Unsurprisingly, there is no trace of narrowband tone noise in the noise spectra and a complete tonal suppression has been achieved. The overall noise performance for this type of poro-serrated trailing edge is also not considered the best.

The poro-serrated trailing edge that produces the best overall noise performance is when the flow resistivity at the sawtooth gaps tunes to about $10 \mathrm{kN} . \mathrm{s} . \mathrm{m}^{-4}$. This optimised flow resistivity value at the sawtooth gaps ensures that not only it is just enough to inhibit the formation of large scale vortex shedding on the sawtooth roots (thus suppressing the vortex shedding tone), but it is also "porous" enough to retain, or even enhance the serration effect. Another interesting outcome is that this particular poro-serrated trailing edge seems to achieve a slightly higher level of broadband noise reduction $(\sim 0.5 \mathrm{~dB}$ in the NPM and up to $1.5 \mathrm{~dB}$ in the $\Delta \mathrm{SPL})$ than the conventional nonflat plate serrated trailing edge.

The flow measurement on the very near wake also reveals several interesting points. First, compared to the baseline straight trailing edge, it has been demonstrated that adding porous foams to the sawtooth gaps of the poroserrated trailing edge will not increase the overall drag coefficient at low blade loading or angle of attack. In addition, the current data shows that a poro-serrated trailing edge can even produce a slightly lower $C_{D(\text { mean })}$ than the baseline straight trailing edge. The $C_{D(\mathrm{rms})}$ produced by the poro-serrated trailing edge is indeed larger than the baseline straight one, but the effect is small and will not change much the overall drag force.

Second, turbulent velocity profiles at the very near wake region were examined at three spanwise locations: sawtooth tip, half-way point of the oblique edge (also called the mid-point) and sawtooth root. Compared to the straight trailing edge, both the nonflat plate serrated and poro-serrated trailing edges show that: (1) their shear layers are lifted up, where their maxima contain higher turbulence intensity, (2) the centreline of their wake profiles contains lower turbulence intensity, and (3) their turbulent velocity profiles at the sawtooth tip are generally similar in character to those at the sawtooth mid-point and root regions. Concerning the third point, the sawtooth tip has exactly the same aerofoil cross section as the baseline straight trailing edge. Therefore, without any external influences, the boundary layer and wake developments there should be the same for either the straight, nonflat plate serrated or poro-serrated trailing edges. The lack of such correlation in the turbulent velocity profiles may indicate that there must be an influence of cross flow in a direction from the sawtooth root to the tip, most probably obliquely, to alter the boundary layer and wake characteristics at the sawtooth tip.

\section{Conclusion}

The flow dynamics within the sawtooth gaps, which was subjected to a wide range of flow resistance in this study (including zero flow resistance as the nonflat plate serrated case), can affect the noise performance of an aerofoil in the following ways:

1. For very low flow resistivity at the sawtooth gap (e.g. the $S 2$ serrated aerofoil),

a. the serration effect is enhanced by the oblique cross flow in a direction from the sawtooth root to tip. The turbulence intensity maxima of the shear layer are lifted away from the wall, while the near wall sublayer attains lower turbulence intensity at the sawtooth tip, mid-point and root than 
those obtained in a straight trailing edge. Substantial broadband noise reduction can be achieved, presumably due to the weaker noise scattering at these locations.

b. The longitudinal transverse flow (vortex shedding) is prominent within the sawtooth gap, which leads to large tone noise radiation.

2. For very high flow resistivity at the sawtooth gap (e.g. the $S 2^{I V}$ poro-serrated aerofoil),

a. the serration effect diminishes (more like a straight trailing edge now), which leads to reduced efficiency in the broadband noise reduction.

b. the transverse flow at the sawtooth gap can be completely suppressed, eventually leads to large tonal noise reduction.

3. For an optimal flow resistivity at the sawtooth gap (e.g. the $S 2^{I I}$ poro-serrated aerofoil),

a. The serration effect is retained, if not slightly enhanced (see Fig. 10) where a further $1.5 \mathrm{~dB}$ in $\Delta \mathrm{SPL}$, or $0.5 \mathrm{~dB}$ in NPM on top of the $S 2$ serrated aerofoil can be achieved. Multiple broadband noise reduction mechanisms might occur in this case (serration + porous material), but it is quite likely that the flow dynamics within the porous material is enhancing the serration, rather than the porous material exerting the "porosity" effect per se.

b. The transverse flow at the sawtooth gap can still be completely suppressed. No tonal noise radiation.

Finally, analysis of the wake velocity for the mean and fluctuating components suggests that the overall drag force is unlikely to increase when a poro-serrated trailing edge is used.

\section{Acknowledgments}

The authors would like to thank the financial support from the EPSRC Impact Acceleration Account - Readiness in the United Kingdom.

\section{References}

[1] Oerlemans, S. and Schepers, G., "Prediction of Wind Turbine Noise Directivity and Swish," $3^{\text {rd }}$ International Meeting on Wind Turbine Noise, 2009, Aalborg, Denmark.

[2] Dassen, T., Parchen, R., Bruggeman, J. and Hagg, F., "Results of a Wind Tunnel Study on the Reduction of Airfoil Self-Noise by the Application of Serrated Blade Trailing Edges," Proceedings of the European Wind Energy Conference, 1996.

[3] Oerlemans, S., Fisher, M., Maeder, T. and Korler, K., "Reduction of Wind Turbine Noise using Optimized Airfoils and Trailing Edge Serrations," AIAA Journal, Vol. 47, 2009, pp. 1470-1481.

[4] Jones, L. E. and Sandberg, R. D., "Direct Numerical Simulations of noise Generated by the Flow over an Airfoil with Trailing Edge Serrations," 15th AIAA/CEAS Aeroacoustic Conference and Exhibit, AIAA Paper 2009-3195, 2009, Miami, Florida.

[5] Gruber, M., Joseph, P. F. and Chong, T. P., "Experimental Investigation of Airfoil Self Noise and Turbulent Wake Reduction by the use of Trailing Edge Serrations," $16^{\text {th }}$ AIAA/CEAS Aeroacoustic Conference and Exhibit, AIAA Paper 2010-3803, 2010, Stockholm, Sweden.

[6] Moreau, D. J. and Doolan C. J., "Noise-Reduction Mechanism of a Flat-Plate Serrated Trailing Edge,” AIAA Journal, Vol. 51, 2013, pp. 2513-2522.

[7] Hurault, J., Gupta, A., Sloth, E., Nielsen, N. C., Borgoltz, A. and Ravetta, P., "Aeroacoustic Wind Tunnel Experiment for Serration Design Optimisation and its Application to a Wind Turbine Rotor," In 6th International Meeting on Wind Turbine Noise, 2015, Glasgow.

[8] Chong, T. P. and Joseph, P. F., "An Experimental Study of Airfoil Instability Tonal Noise with Trailing Edge Serrations," Journal of Sound and Vibration, Vol. 332 2013, pp. 6335-6358.

[9] Chong, T. P., Vathylakis, A., Joseph, P. F. and Gruber, M., "Self-Noise Produced by an Airfoil with Nonflat plate Trailing-Edge Serrations,” AIAA Journal, Vol. 51, 2013, pp. 2665-2677.

[10] Chong, T. P., Joseph, P. F. and Gruber, M., "Airfoil Self Noise Reduction by Non-Flat plate type Trailing Edge Serrations," Applied Acoustics, Vol. 74, 2013, pp. 607-613.

[11] Vathylakis, A, Chong, T. P. and Joseph, P. F., "Poro-Serrated Trailing-Edge Devices for Airfoil Self-Noise Reduction,” AIAA Journal, Vol. 53, 2015, pp. 3379-3394.

[12] Howe, M. S., "Noise Produced by a Sawtooth Trailing Edge," Journal of Acoustical Society of America, Vol. 90, 1991 , pp. $482-487$.

[13] Lyu, B., Azarpeyvand, M. and Sinayoko, S, "A Trailing-Edge Noise Model for Serrated Edges," $21^{\text {st }}$ AIAA/CEAS Aeroacoustic Conference and Exhibit, AIAA Paper 2015-2362, 2015, Dallas, Texas.

[14] Chong, T. P. and Vathylakis, A., "On the Aeroacoustic and Flow Structures Developed on a Flat Plate with a Serrated Sawtooth Trailing Edge," Journal of Sound and Vibration, Vol. 354, 2015, pp. 65-90.

[15] Geyer, T., Sarradj, E. and Fritzsche, C., "Measurement on the Noise Generation at the Trailing Edge of Porous Airfoils," Experiments in Fluids, Vol. 48, 2010, pp. 291-308.

[16] Herr, M., Rossignol, K. S., Delfs, J., Mößner, M. and Lippitz, N., "Specifications of Porous Materials for Low-Noise Trailing-Edge Applications," $20^{\text {th }}$ AIAA/CEAS Aeroacoustic Conference and Exhibit, AIAA Paper 2014-3041, 2014, Atlanta, Georgia. 
[17] Vathylakis, A., Chong, T. P. and Kim, J. H., "Design of a Low-Noise Aeroacoustic Wind Tunnel Facility at Brunel University," $20^{\text {th }}$ AIAA/CEAS Aeroacoustic Conference and Exhibit, AIAA Paper 2014-3288, 2014, Atlanta, Georgia.

[18] Hah, C. and Lakshminarayana, B., "Measurement and Prediction of Mean Velocity and Turbulence Structure in the Near Wake of an Airfoil," Journal of Fluid Mechanics, Vol. 115, 1982, pp. 251-282. 